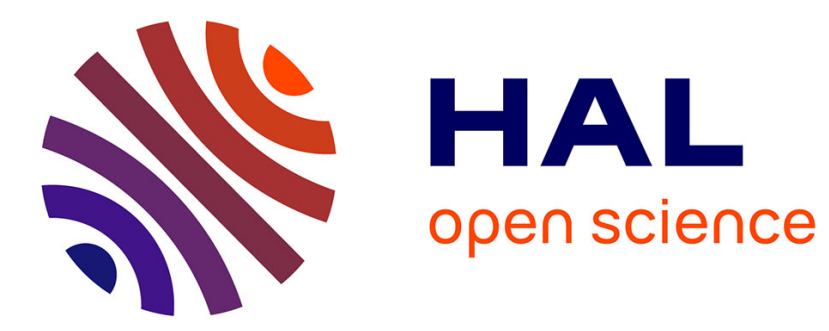

\title{
Multiscale Modeling of Imperfect Interfaces and Applications
}

Serge Dumont, Frédéric Lebon, Maria Letizia Raffa, Raffaella Rizzoni, Hélène Welemane

\section{- To cite this version:}

Serge Dumont, Frédéric Lebon, Maria Letizia Raffa, Raffaella Rizzoni, Hélène Welemane. Multiscale Modeling of Imperfect Interfaces and Applications. A. Ibrahimbegovic. Computational Methods for Solids and Fluids, 41, Springer International Publishing, pp.81-122, 2016, Computational Methods in Applied Sciences, 978-3-319-27994-7. 10.1007/978-3-319-27996-1_5 . hal-01711672

\section{HAL Id: hal-01711672 \\ https://hal.science/hal-01711672}

Submitted on 26 Feb 2018

HAL is a multi-disciplinary open access archive for the deposit and dissemination of scientific research documents, whether they are published or not. The documents may come from teaching and research institutions in France or abroad, or from public or private research centers.
L'archive ouverte pluridisciplinaire HAL, est destinée au dépôt et à la diffusion de documents scientifiques de niveau recherche, publiés ou non, émanant des établissements d'enseignement et de recherche français ou étrangers, des laboratoires publics ou privés. 


\section{Multiscale Modeling of Imperfect Interfaces and Applications}

Chapter · February 2016

DOI: 10.1007/978-3-319-27996-1_5 


\title{
Multiscale Modeling of Imperfect Interfaces and Applications
}

\author{
S. Dumont, F. Lebon, M.L. Raffa, R. Rizzoni and H. Welemane
}

\begin{abstract}
Modeling interfaces between solids is of great importance in the fields of mechanical and civil engineering. Solid/solid interface behavior at the microscale has a strong influence on the strength of structures at the macroscale, such as gluing, optical systems, aircraft tires, pavement layers and masonry, for instance. In this lecture, a deductive approach is used to derive interface models, i.e. the thickness of the interface is considered as a small parameter and asymptotic techniques are introduced. A family of imperfect interface models is presented taking into account cracks at microscale. The proposed models combine homogenization techniques for microcracked media both in three-dimensional and two-dimensional cases, which leads to a cracked orthotropic material, and matched asymptotic method. In particular, it is shown that the Kachanov type theory leads to soft interface models and, alternatively,
\end{abstract}

S. Dumont

Université de Nîmes Place Gabriel Péri, 30000 Nîmes, France

S. Dumont

Laboratoire de Mécanique et d'Acoustique (LMA) - CNRS UPR 7051, 4 Impasse

Nikola Tesla, CS 40006-13453 Marseille Cedex 13, France

F. Lebon $(\varangle)$

Laboratoire de Mécanique et d'Acoustique (LMA) - CNRS UPR 7051,

Aix-Marseille University, Centrale Marseille, 4 Impasse Nikola Tesla,

CS 40006 - 13453 Marseille Cedex 13, France

e-mail: lebon@lma.cnrs-mrs.fr

M.L. Raffa

Department of Civil Engineering and Computer Science Engineering (DICII),

University of Rome "Tor Vergata", via del Politecnico 1 - 00133, Rome, Italy

M.L. Raffa

Laboratoire de Mécanique et d'Acoustique (LMA) - CNRS UPR 7051,

Aix-Marseille University, 4 Impasse Nikola Tesla CS 40006 - 13453,

Marseille Cedex 13, France

R. Rizzoni

Department of Engineering, University of Ferrara, Via Saragat 1, 44122, Ferrara, Italy

H. Welemane

University of Toulouse, National Engineering School of Tarbes,

47, avenue d'Azereix, BP 1629, 65016 Tarbes Cedex, France

(C) Springer International Publishing Switzerland 2016

A. Ibrahimbegovic (ed.), Computational Methods for Solids and Fluids,

Computational Methods in Applied Sciences 41,

DOI 10.1007/978-3-319-27996-1_5 
that Goidescu et al. theory leads to stiff interface models. A fully nonlinear variant of the model is also proposed, derived from the St. Venant-Kirchhoff constitutive equations. Some applications to elementary masonry structures are presented.

\section{Introduction}

The study of interfaces between deformable solids significantly developed thanks to the rising interest of scientists and industries in mechanics of composite materials. Those first studies, in particular, focused on the presence of matrix-fiber interfaces in composite media and their effect on the determination of the effective thermoelastic properties of this kind of materials. Within the framework of these theories on mechanical behavior of composites, a commonly adopted assumption was the requirement of the continuity in terms of stresses and displacements at the interfaces among the principal constituents. The stress-based interface condition origins from the local equilibrium and the displacement-based interface condition derives from the hypothesis of perfect bonding. Such an interface condition was defined as perfect interface. Nevertheless, the assumption of perfect interfaces is established to be inappropriate in many mechanical problems. Indeed, the interface between two bodies or two parts of a body, defined as adherents, is a favorable zone to complex physico-chemical reactions and vulnerable to mechanical damage. Goland and Reissner [22], in the forties, were surely the first to model a thin adhesive as a weak interface, i.e. they were the first to assume that the adherents were linked by a continuous distribution of springs. Such an interface, is defined as spring type. Goland and Reissner have noted that the thinness suggests to consider constant stresses in the adhesive, and some years later, Gilibert and Rigolot [19] found a rational justification of this fact by means of the asymptotic expansion method, assuming that the thickness and the elastic properties of the adhesive had the same order of smallness $\varepsilon$. During the eighties and nineties, the relaxation of the perfect interface approximation was largely investigated, aiming principally to applicate these theories to composite materials with coated fibers or particles $[3,26]$, or in the case of decohesion and nucleation problems in cohesive zones [44, 45]. One of the first definition of imperfect interface was certainly due to Hashin and Benveniste [3, 26]. Particularly, Hashin concentrates his research in the case of composite material with thin layer or coating enveloping its reinforcing constituents (fibers). Such an interfacial layer is generally referred to as interphase, and its presence can be due to chemical interaction between the constituents or it may be introduced by design aiming to improve the mechanical properties of the composite. Several investigations in literature, before and after the work of Hashin, modeled this kind of problem with the so-called three-phase-material theory. Such a description requires, obviously, the knowledge of the interphase properties. These constitutive informations are rarely available, primarily, because the interphase material properties are in situ properties which are not necessarily equal to those of the bulk material, and additionally, the interphase may vary within a transition zone from one constituent to another. 
Accordingly, in most cases, the interphase properties are unmeasurable. The Imperfect interface theory was formulated by Hashin [26-29] in order to overcome these challenges. This alternative model was based to the main idea that if the interphase has significant effects on the overall response, then its properties must be significantly different from those of the constituents, in general, much more flexible. To this aim, the attempts for explicit modeling of the three-dimensional interphase are highly reduced by replacing it with a two-dimensional imperfect interface. In particular, within the Hashin imperfect interface model [26] the discontinuity in terms of displacements is allowed, instead, the continuity in terms of stresses, according local equilibrium, is preserved. Hashin [26], as Goland [22] before him, made the simplest assumption that the displacement discontinuity is linearly proportional to the traction vector:

$$
\boldsymbol{\sigma}^{\left(\Omega_{1}\right)} \mathbf{n}=\boldsymbol{\sigma}^{\left(\Omega_{2}\right)} \mathbf{n}=\mathbf{D}[\mathbf{u}] \quad[\mathbf{u}]=\mathbf{u}^{\left(\Omega_{1}\right)}-\mathbf{u}^{\left(\Omega_{2}\right)}
$$

where $\boldsymbol{\sigma}^{\left(\Omega_{1}\right),\left(\Omega_{2}\right)} \mathbf{n}$ is the interfacial stress vector relative to the solids $\Omega_{1}$ and $\Omega_{2}$ in contact; $[\mathbf{u}]$ and $\mathbf{u}^{\left(\Omega_{1}\right),\left(\Omega_{2}\right)}$ are the displacement jump vector and displacement vector, respectively; $\mathbf{D}$ is a matrix which contains the spring constant type material parameters, in normal and tangential directions; these latter have dimension of stress divided by length. In the following, these parameters are referred as interface stiffnesses. It is worth to point out that infinite values of the interface stiffnesses imply vanishing of displacement jumps and therefore perfect interface conditions. At the other asymptotic limit, zero values of the stiffnesses imply vanishing of interface tractions and therefore, debonding conditions. Any finite positive values of the interface stiffnesses define an imperfect interface.

Hashin, with his pioneering work, determined the effective elastic properties and thermal expansions coefficients both for unidirectional fiber composites with imperfect interfaces conditions [26] and for composites with spherical inclusions and particles [27, 28]. Moreover, he demonstrated that the three-phase-material approach was a special case of the imperfect interface theory. It is worth remarking that Hashin, as first, showed that the interface stiffnesses (he referred them as interface parameters) can be simply related to the interphase properties and geometry [26].

Hashin and Benveniste, independently, generalized the classical extremum principles of the theory of elasticity for composite bodies to the case of an imperfect interface described by linear relations between interface displacement jumps and tractions [3, 29].

In the work of Bövik [9], the idea to use a simple tool that is the Taylor expansion of the relevant physical fields in a thin interphase, combined with the use of surface differential operators on a curved surface, has been applied to achieve the representation of a thin interphase by an imperfect interface. The idea of a Taylor expansion was also adopted by Hashin to derive the spring-type interface model for soft elastic interfaces [28] and for interphases of arbitrary conductivity and elastic moduli [30]. More recently, $\mathrm{Gu}$ [23, 24] derived an imperfect interface model for coupled multifield phenomena (thermal conductivity, elasticity and piezoelectricity) 
by applying Taylor expansion to an arbitrarily curved thin interphase between two adjoining phases; he also introduced some new coordinate-free interfacial operators.

All the above cited imperfect interface models are derived by assuming an isotropic interphase.

In a quite recent work Benveniste [4], provided a generalization of the Bövik model to an arbitrarily curved three-dimensional thin anisotropic layer between two anisotropic media. Benveniste model is carried out in the setting of unsteady heat conduction and dynamic elasticity. The derived interface model consists of expressions for the jumps in the physical fields, i.e. temperature and normal heat flux in conduction, and displacements and tractions in elasticity, across the interface.

Additionally, derivations of spring-type interface models by using asymptotic methods, for different geometrical configurations, have been given, among other by Klarbring [36, 37] and Geymonat [18].

A much less studied imperfect interface condition is the one obtained starting from a stiff and thin interphase, the so called stiff interface (or equivalently hard interface). Differently from the soft case, the hard interface is characterized by a jump of the traction vector across the interface and by continuity of displacements. Benveniste and Miloh [5], generalizing the study made by Caillerie [10] for curved interfaces, demonstrate that depending on its degree of stiffness with respect to the bodies in contact, a stiff thin interphase translates itself into a much richer class of imperfect interfaces than a soft interphase does. Within their study, a thin curved isotropic layer of constant thickness between two elastic isotropic media in a twodimensional plane strain setting, is considered. The properties of the curved layer are allowed to vary in the tangential direction. It is shown that depending on the softness or stiffness of the interphase with respect to the neighboring media, as determined by the magnitude of the non-dimensional Lamé parameters $\bar{\lambda}_{c}$ and $\bar{\mu}_{c}$, there exists seven distinct regimes of interface conditions according the following expressions:

$$
\bar{\lambda}_{i}=\frac{\tilde{\lambda}_{i}}{\varepsilon^{N}} \quad \bar{\mu}_{i}=\frac{\tilde{\mu}_{i}}{\varepsilon^{N}}
$$

where $\tilde{\lambda}_{c}$ and $\tilde{\mu}_{c}$ are non-dimensional constant Lamé parameters of the material interphase, $\varepsilon$ in the non-dimensional interphase thickness and $N$ is a negative or positive integer or zero. Accordingly with the above definition these regimes may be distinguished in: (a) vacuous contact type interface for $N \leqslant-2$, (b) spring type interface for $N=-1$, (c) ideal contact type interface for $N=0$, (d) membrane type interface for $N=1$, (e) inextensible membrane type interface for $N=2$, (f) inextensible shell type interface for $N=3,(\mathrm{~g})$ rigid contact type interface for $N \geqslant 4$. The first two conditions are characteristic of a soft interphase whereas the last four are characteristic of a stiff interphase. The cases (a), (c) and (g) are the classical ones: in case (a) the tractions vanish (debonding), in case (c) the displacements and tractions are continuous (perfect interface condition), and in case (g) the displacements vanish. Benveniste and Miloh [5], for the first time, derived the interface conditions for the hard cases (d), (e) and (f), by applying a formal asymptotic expansion for the displacements and stresses fields in the thin layer interphase. 
In the present chapter, two kind of imperfect interface conditions are essentially referred to: the soft interphase case which brings to a spring-type, both linear and nonlinear, interface, and the hard interphase case which brings to a more general interface model that includes, as will be shown in the next section, the perfect interface conditions. In order to make an analogy with the Benveniste's classifications, the cases with $N=-1$ and $N=0$ will be analyzed. It is worth remarking some differences between the hard interface case considered in this work and that defined by Benveniste and Miloh for $N=0$. In fact in the work, the case $N=0$, according formers papers $[14,38,39,52,53]$ will be studied within the framework of higher order theory. This choice, extensively detailed in the following, leads to the evidence that the case $N=0$ is an effective imperfect interface condition, i.e. stress vector and displacement jump vector in the one-order formulation have been found to be non-null. As a result, the perfect interface has been established to be a particular case of the hard interface condition at the zero-order [53], in what follows this evidence will be analytically derived within the asymptotic framework.

The imperfect interface models, object of the present chapter, are consistently derived by coupling a homogenization approach for microcracked media under the non-interacting approximation (NIA) [21, 34, 35, 57, 60], and arguments of asymptotic analysis [38, 39, 52, 53]. Such a method, is defined imperfect interface approach.

The text is organized as follows: Sects. 2 and 3 are devoted to detail the framework of the imperfect interface approach and to enforce it in order to derive several interface models, particularly, in Sect. 3 a nonlinear imperfect interface model is presented; Sect. 4 is consecrated to a simple numerical application useful to validate these interface models; finally, in Sect. 5 some conclusions are outlined.

\section{Imperfect Interface Approach}

In this section, it is shown how matched asymptotic expansion method coupled together an homogenization technique for microcracked media, give birth to both soft and hard imperfect interface laws.

\subsection{Matched Asymptotic Expansion Method}

It is worth pointing out that the application of the asymptotic methods to obtain governing equations of imperfect interface starting from thin layer problems in the elasticity framework, has a consistent mathematical background [10-13, 26-28, 30, $55,56]$. Ould Khaoua among other, in his doctoral thesis [46], studied the elastic equilibrium problem $\mathscr{P}^{\varepsilon}$ under the hypothesis of small perturbations. The author demonstrates that the solution of the reference problem (i.e. with an elastic thin layer of thickness $\varepsilon$ ) $\mathscr{P}^{\varepsilon}$, that is expressed in terms of both stress and displacement fields 
$\left(\boldsymbol{\sigma}^{\varepsilon}, \mathbf{u}^{\varepsilon}\right)$, for $\varepsilon \rightarrow 0$ admits a limit $(\boldsymbol{\sigma}, \mathbf{u})$ and that this limit solution is also the solution of the limit problem $\mathscr{P}\left(\mathscr{P}^{\varepsilon} \rightarrow \mathscr{P}\right.$ for $\left.\varepsilon \rightarrow 0\right)$. Additionally, Ould Khaoua [46] has found, as Hashin [26] before, that the mechanical and geometrical characteristics of the layer (interphase) are retained in the interface stiffnesses of the soft interface governing equations.

The matched asymptotic expansion method [38-40, 52, 53], adopted in this work, is detailed in the following paragraphs.

\subsubsection{General Notations}

With reference to [53], let the problem general notations be detailed. A thin layer $\mathscr{B}^{\varepsilon}$ with cross-section $\mathscr{S}$ and uniform small thickness $\varepsilon \ll 1$ is considered, $\mathscr{S}$ being an open bounded set in $\mathbb{R}^{2}$ with a smooth boundary. In the following $\mathscr{B}^{\varepsilon}$ and $\mathscr{S}$ will be called interphase and interface, respectively. The interphase lies between two bodies, named as adherents, occupying the reference configurations $\Omega^{\varepsilon} \pm \subset \mathbb{R}^{3}$. In such a way, the interphase represents the adhesive joining the two bodies $\Omega_{+}^{\varepsilon}$ and $\Omega_{-}^{\varepsilon}$. Let $\mathscr{S}^{\varepsilon}{ }_{ \pm}$be taken to denote the plane interfaces between the interphase and the adherents and let $\Omega^{\varepsilon}=\Omega^{\varepsilon}{ }_{ \pm} \cup \mathscr{S}^{\varepsilon}{ }_{ \pm} \cup \mathscr{B}^{\varepsilon}$ denote the composite system comprising the interphase and the adherents.

It is assumed that the adhesive and the adherents are perfectly bonded and thus, the continuity of the displacement and stress vector fields across $\mathscr{S}^{\varepsilon}{ }_{ \pm}$is ensured.

An orthonormal Cartesian basis $\left(O, \mathbf{i}_{1}, \mathbf{i}_{2}, \mathbf{i}_{3}\right)$ is introduced and let $\left(x_{1}, x_{2}, x_{3}\right)$ be taken to denote the three coordinates of a particle. The origin lies at the center of the interphase midplane and the $x_{3}$-axis runs perpendicular to the open bounded set $\mathscr{S}$, as illustrated in Fig. 1.

The materials of the composite system are assumed to be homogeneous and initially linearly elastic and let $\mathbb{A}_{ \pm}, \mathbb{B}^{\varepsilon}$ be the fourth-rank elasticity tensors of the

(a)

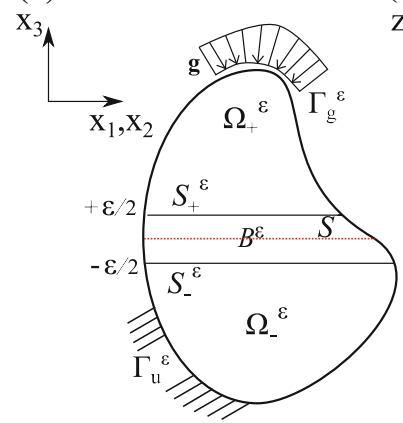

(b)

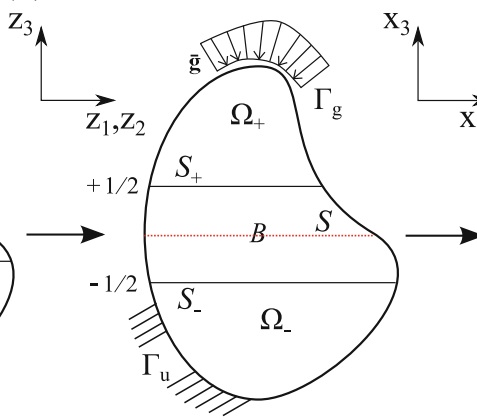

(c)

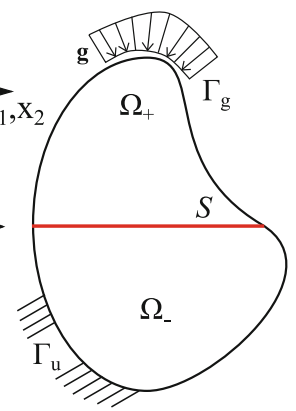

Fig. 1 Asymptotic procedure-Synoptic sketch of three steps performed in the matched asymptotic expansion approach: a the reference configuration with the $\varepsilon$-thick interphase; $\mathbf{b}$ the rescaled configuration; $\mathbf{c}$ the final configuration with the zero-thick interface 
adherents and of the interphase, respectively. The tensors $\mathbb{A}_{ \pm}, \mathbb{B}^{\varepsilon}$ are assumed to be symmetric, with the minor and major symmetries, and positive definite. The adherents are subjected to a body force density $\mathbf{f}^{ \pm}: \Omega^{\varepsilon}{ }_{ \pm} \mapsto \mathbb{R}^{3}$ and to a surface force density $\mathbf{g}^{ \pm}: \Gamma_{g}^{\varepsilon} \mapsto \mathbb{R}^{3}$ on $\Gamma_{g}^{\varepsilon} \subset\left(\partial \Omega^{\varepsilon}+\backslash \mathscr{S}_{+}^{\varepsilon}\right) \cup\left(\partial \Omega_{-}^{\varepsilon} \backslash \mathscr{S}_{-}^{\varepsilon}\right)$. Body forces are neglected in the adhesive.

On $\Gamma_{u}^{\varepsilon}=\left(\partial \Omega^{\varepsilon}+\backslash \mathscr{S}_{+}^{\varepsilon}\right) \cup\left(\partial \Omega^{\varepsilon}{ }_{-} \backslash \mathscr{S}_{-}^{\varepsilon}\right) \backslash \Gamma_{g}^{\varepsilon}$, homogeneous boundary conditions are prescribed:

$$
\mathbf{u}^{\varepsilon}=\mathbf{0} \text { on } \Gamma_{u}^{\varepsilon},
$$

where $\mathbf{u}^{\varepsilon}: \Omega^{\varepsilon} \mapsto \mathbb{R}^{3}$ is the displacement field defined on $\Omega^{\varepsilon} . \Gamma_{g}^{\varepsilon}, \Gamma_{u}^{\varepsilon}$ are assumed to be located far from the interphase, in particular, the external boundaries of the interphase $\mathscr{B}^{\varepsilon}\left(\partial \mathscr{S} \times\left(-\frac{\varepsilon}{2}, \frac{\varepsilon}{2}\right)\right)$ are assumed to be stress-free. The fields of the external forces are endowed with sufficient regularity to ensure the existence of equilibrium configuration.

\subsubsection{Rescaling Process}

The rescaling phase in the asymptotic process represents a mathematical construct [12], not a physically-based configuration of the studied composed system. This standard step is used in the asymptotic expansion method, in order to eliminate the dependency on the small parameter $\varepsilon$ of the integration domains. This rescaling procedure can be seen as a change of spatial variables $\hat{\mathbf{p}}:\left(x_{1}, x_{2}, x_{3}\right) \rightarrow\left(z_{1}, z_{2}, z_{3}\right)$ in the interphase [12]:

$$
z_{1}=x_{1}, \quad z_{2}=x_{2}, \quad z_{3}=\frac{x_{3}}{\varepsilon}
$$

resulting

$$
\frac{\partial}{\partial z_{1}}=\frac{\partial}{\partial x_{1}}, \quad \frac{\partial}{\partial z_{2}}=\frac{\partial}{\partial x_{2}}, \quad \frac{\partial}{\partial z_{3}}=\varepsilon \frac{\partial}{\partial x_{3}} .
$$

Moreover, in the adherents the following change of variables $\overline{\mathbf{p}}:\left(x_{1}, x_{2}, x_{3}\right) \rightarrow$ $\left(z_{1}, z_{2}, z_{3}\right)$ is also introduced:

$$
z_{1}=x_{1}, \quad z_{2}=x_{2}, \quad z_{3}=x_{3} \pm \frac{1}{2}(1-\varepsilon)
$$

where the plus (minus) sign applies whenever $x \in \Omega^{\varepsilon}+\left(x \in \Omega^{\varepsilon}{ }_{-}\right)$, with

$$
\frac{\partial}{\partial z_{1}}=\frac{\partial}{\partial x_{1}}, \quad \frac{\partial}{\partial z_{2}}=\frac{\partial}{\partial x_{2}}, \quad \frac{\partial}{\partial z_{3}}=\frac{\partial}{\partial x_{3}}
$$

After the change of variables (2), the interphase occupies the domain

$$
\mathscr{B}=\left\{\left(z_{1}, z_{2}, z_{3}\right) \in \mathbb{R}^{3}:\left(z_{1}, z_{2}\right) \in \mathscr{S},\left|z_{3}\right|<\frac{1}{2}\right\}
$$


and the adherents occupy the domains $\Omega_{ \pm}=\Omega^{\varepsilon}{ }_{ \pm} \pm \frac{1}{2}(1-\varepsilon) \mathbf{i}_{3}$, as shown in Fig. 1 . The sets $\mathscr{S}_{ \pm}=\left\{\left(z_{1}, z_{2}, z_{3}\right) \in \mathbb{R}^{3}:\left(z_{1}, z_{2}\right) \in \mathscr{S}, z_{3}= \pm \frac{1}{2}\right\}$ are taken to denote the interfaces between $\mathscr{B}$ and $\Omega_{ \pm}$and $\Omega=\Omega_{+} \cup \Omega_{-} \cup \mathscr{B} \cup \mathscr{S}_{+} \cup \mathscr{S}_{-}$is the rescaled configuration of the composite body. Lastly, $\Gamma_{u}$ and $\Gamma_{g}$ indicates the images of $\Gamma_{u}^{\varepsilon}$ and $\Gamma_{g}^{\varepsilon}$ under the change of variables, and $\overline{\mathbf{f}}^{ \pm}:=\mathbf{f}^{ \pm} \circ \overline{\mathbf{p}}^{-1}$ and $\overline{\mathbf{g}}^{ \pm}:=\mathbf{g}^{ \pm} \circ \overline{\mathbf{p}}^{-1}$ the rescaled external forces.

\subsubsection{Concerning Kinematics}

Following the approach proposed by [38, 53], let focus on the kinematics of the elastic problem. After taking $\hat{\mathbf{u}}^{\varepsilon}=\mathbf{u}^{\varepsilon} \circ \hat{\mathbf{p}}^{-1}$ and $\overline{\mathbf{u}}^{\varepsilon}=\mathbf{u}^{\varepsilon} \circ \overline{\mathbf{p}}^{-1}$ to denote the displacement fields from the rescaled adhesive and adherents, respectively, the asymptotic expansions of the displacement fields with respect to the small parameter $\varepsilon$ take the form:

$$
\begin{aligned}
& \mathbf{u}^{\varepsilon}\left(x_{1}, x_{2}, x_{3}\right)=\mathbf{u}^{0}+\varepsilon \mathbf{u}^{1}+\varepsilon^{2} \mathbf{u}^{2}+o\left(\varepsilon^{2}\right) \\
& \hat{\mathbf{u}}^{\varepsilon}\left(z_{1}, z_{2}, z_{3}\right)=\hat{\mathbf{u}}^{0}+\varepsilon \hat{\mathbf{u}}^{1}+\varepsilon^{2} \hat{\mathbf{u}}^{2}+o\left(\varepsilon^{2}\right) \\
& \overline{\mathbf{u}}^{\varepsilon}\left(z_{1}, z_{2}, z_{3}\right)=\overline{\mathbf{u}}^{0}+\varepsilon \overline{\mathbf{u}}^{1}+\varepsilon^{2} \overline{\mathbf{u}}^{2}+o\left(\varepsilon^{2}\right)
\end{aligned}
$$

Interphase:

The displacement gradient tensor of the field $\hat{\mathbf{u}}^{\varepsilon}$ in the rescaled interphase is computed as:

$$
\hat{\mathbf{H}}=\varepsilon^{-1}\left[\begin{array}{ll}
0 & \hat{u}_{\alpha, 3}^{0} \\
0 & \hat{u}_{3,3}^{0}
\end{array}\right]+\left[\begin{array}{cc}
\hat{u}_{\alpha, \beta}^{0} & \hat{u}_{\alpha, 3}^{1} \\
\hat{u}_{3, \beta}^{0} & \hat{u}_{3,3}^{1}
\end{array}\right]+\varepsilon\left[\begin{array}{cc}
\hat{u}_{\alpha, \beta}^{1} & \hat{u}_{\alpha, 3}^{2} \\
\hat{u}_{3, \beta}^{1} & \hat{u}_{3,3}^{2}
\end{array}\right]+O\left(\varepsilon^{2}\right)
$$

where $\alpha=1,2$, so that the strain tensor can be obtained as:

$$
\mathbf{e}\left(\hat{\mathbf{u}}^{\varepsilon}\right)=\varepsilon^{-1} \hat{\mathbf{e}}^{-1}+\hat{\mathbf{e}}^{0}+\varepsilon \hat{\mathbf{e}}^{1}+O\left(\varepsilon^{2}\right)
$$

with:

$$
\begin{aligned}
& \hat{\mathbf{e}}^{-1}=\left[\begin{array}{cc}
0 & \frac{1}{2} \hat{u}_{\alpha, 3}^{0} \\
\frac{1}{2} \hat{u}_{\alpha, 3}^{0} & \hat{u}_{3,3}^{0}
\end{array}\right]=\operatorname{Sym}\left(\hat{\mathbf{u}}_{, 3}^{0} \otimes \mathbf{i}_{3}\right) \\
& \hat{\mathbf{e}}^{k}=\left[\begin{array}{cc}
\operatorname{Sym}\left(\hat{u}_{\alpha, \beta}^{k}\right) & \frac{1}{2}\left(\hat{u}_{3, \alpha}^{k}+\hat{u}_{\alpha, 3}^{k+1}\right) \\
\frac{1}{2}\left(\hat{u}_{3, \alpha}^{k}+\hat{u}_{\alpha, 3}^{k+1}\right) & \hat{u}_{3,3}^{k+1}
\end{array}\right]=\operatorname{Sym}\left(\hat{\mathbf{u}}_{, 1}^{k} \otimes \mathbf{i}_{1}+\hat{\mathbf{u}}_{, 2}^{k} \otimes \mathbf{i}_{2}+\hat{\mathbf{u}}_{, 3}^{k+1} \otimes \mathbf{i}_{3}\right)
\end{aligned}
$$


where $\operatorname{Sym}(\cdot)$ gives the symmetric part of the enclosed tensor and $k=0,1$, and $\otimes$ is the dyadic product between vectors such as: $(\mathbf{a} \otimes \mathbf{b})_{i j}=a_{i} b_{j}$. Moreover, the following notation for derivatives is adopted: $f_{, j}$ denoting the partial derivative of $f$ with respect to $z_{j}$.

\section{Adherents:}

The displacement gradient tensor of the field $\overline{\mathbf{u}}^{\varepsilon}$ in the adherents is computed as:

$$
\overline{\mathbf{H}}=\left[\begin{array}{ll}
\bar{u}_{\alpha, \beta}^{0} & \bar{u}_{\alpha, 3}^{0} \\
\bar{u}_{3, \beta}^{0} & \bar{u}_{3,3}^{0}
\end{array}\right]+\varepsilon\left[\begin{array}{cc}
\bar{u}_{\alpha, \beta}^{1} & \bar{u}_{\alpha, 3}^{1} \\
\bar{u}_{3, \beta}^{1} & \bar{u}_{3,3}^{1}
\end{array}\right]+O\left(\varepsilon^{2}\right)
$$

so that the strain tensor can be obtained as:

$$
\mathbf{e}\left(\overline{\mathbf{u}}^{\varepsilon}\right)=\varepsilon^{-1} \overline{\mathbf{e}}^{-1}+\overline{\mathbf{e}}^{0}+\varepsilon \overline{\mathbf{e}}^{1}+O\left(\varepsilon^{2}\right)
$$

with:

$$
\begin{gathered}
\overline{\mathbf{e}}^{-1}=\mathbf{0} \\
\overline{\mathbf{e}}^{k}=\left[\begin{array}{cc}
\operatorname{Sym}\left(\bar{u}_{\alpha, \beta}^{k}\right) & \frac{1}{2}\left(\bar{u}_{3, \alpha}^{k}+\bar{u}_{\alpha, 3}^{k}\right) \\
\frac{1}{2}\left(\bar{u}_{3, \alpha}^{k}+\bar{u}_{\alpha, 3}^{k}\right) & \bar{u}_{3,3}^{k}
\end{array}\right]=\operatorname{Sym}\left(\overline{\mathbf{u}}_{, 1}^{k} \otimes \mathbf{i}_{1}+\overline{\mathbf{u}}_{, 2}^{k} \otimes \mathbf{i}_{2}+\overline{\mathbf{u}}_{, 3}^{k} \otimes \mathbf{i}_{3}\right)
\end{gathered}
$$

with $k=0,1$.

\subsubsection{Concerning Equilibrium}

With reference to the work by [38, 53], the stress fields in the rescaled adhesive and adherents, $\hat{\boldsymbol{\sigma}}^{\varepsilon}=\sigma \circ \hat{\mathbf{p}}^{-1}$ and $\overline{\boldsymbol{\sigma}}^{\varepsilon}=\sigma \circ \overline{\mathbf{p}}^{-1}$ respectively, can be represented as asymptotic expansions:

$$
\begin{aligned}
& \boldsymbol{\sigma}^{\varepsilon}=\boldsymbol{\sigma}^{0}+\varepsilon \boldsymbol{\sigma}^{1}+O\left(\varepsilon^{2}\right) \\
& \hat{\boldsymbol{\sigma}}^{\varepsilon}=\hat{\boldsymbol{\sigma}}^{0}+\varepsilon \hat{\boldsymbol{\sigma}}^{1}+O\left(\varepsilon^{2}\right) \\
& \overline{\boldsymbol{\sigma}}^{\varepsilon}=\overline{\boldsymbol{\sigma}}^{0}+\varepsilon \overline{\boldsymbol{\sigma}}^{1}+O\left(\varepsilon^{2}\right)
\end{aligned}
$$

\section{Equilibrium Equations in the Interphase:}

As body forces are neglected in the adhesive, the equilibrium equation is:

$$
\operatorname{div} \hat{\boldsymbol{\sigma}}^{\varepsilon}=\mathbf{0}
$$


Substituting the representation from (16b) into the equilibrium equation (17) and using (3), it becomes:

$$
\begin{aligned}
0 & =\hat{\sigma}_{i \alpha, \alpha}^{\varepsilon}+\varepsilon^{-1} \hat{\sigma}_{i 3,3}^{\varepsilon} \\
& =\varepsilon^{-1} \hat{\sigma}_{i 3,3}^{0}+\hat{\sigma}_{i \alpha, \alpha}^{0}+\hat{\sigma}_{i 3,3}^{1}+\varepsilon \hat{\sigma}_{i \alpha, \alpha}^{1}+O(\varepsilon)
\end{aligned}
$$

where $\alpha=1,2$. Equation (18) has to be satisfied for any value of $\varepsilon$, leading to:

$$
\begin{aligned}
\hat{\sigma}_{i 3,3}^{0} & =0 \\
\hat{\sigma}_{i 1,1}^{0}+\hat{\sigma}_{i 2,2}^{0}+\hat{\sigma}_{i 3,3}^{1} & =0
\end{aligned}
$$

where $i=1,2,3$.

Equation(19) shows that $\hat{\sigma}_{i 3}^{0}$ is not dependent on $z_{3}$ in the adhesive, and thus it can be written:

$$
\left[\hat{\sigma}_{i 3}^{0}\right]=0
$$

where [•] denotes the jump between $z_{3}=\frac{1}{2}$ and $z_{3}=-\frac{1}{2}$.

In view of (21), Eq. (20), for $i=3$, can be rewritten in the integrated form

$$
\left[\hat{\sigma}_{33}^{1}\right]=-\hat{\sigma}_{13,1}^{0}-\hat{\sigma}_{23,2}^{0}
$$

Equilibrium Equations in the Adherents:

The equilibrium equation in the adherents is:

$$
\operatorname{div} \overline{\boldsymbol{\sigma}}^{\varepsilon}+\overline{\mathbf{f}}=\mathbf{0}
$$

Substituting the representation form (16c) into the equilibrium Eq. (23) and taking into account that it has to be satisfied for any value of $\varepsilon$, it leads to:

$$
\begin{aligned}
& \operatorname{div} \overline{\boldsymbol{\sigma}}^{0}+\overline{\mathbf{f}}=\mathbf{0} \\
& \operatorname{div} \overline{\boldsymbol{\sigma}}^{1}=\mathbf{0}
\end{aligned}
$$

\subsubsection{Matching External and Internal Expansions}

Due to the perfect bonded assumption between $\mathscr{B}^{\varepsilon}$ and $\Omega_{ \pm}^{\varepsilon}$, the continuity conditions at $\mathscr{S}_{ \pm}^{\varepsilon}$ for the fields $\mathbf{u}^{\varepsilon}$ and $\sigma^{\varepsilon}$ lead to matching relationships between external and internal expansions $[38,53]$. In particular, in terms of displacements the following relationship have to be satisfied:

$$
\mathbf{u}^{\varepsilon}\left(\mathbf{x}_{\alpha}, \pm \frac{\varepsilon}{2}\right)=\hat{\mathbf{u}}^{\varepsilon}\left(\mathbf{z}_{\alpha}, \pm \frac{1}{2}\right)=\overline{\mathbf{u}}^{\varepsilon}\left(\mathbf{z}_{\alpha}, \pm \frac{1}{2}\right)
$$


where $\mathbf{x}_{\alpha}:=\left(x_{1}, x_{2}\right), \mathbf{z}_{\alpha}:=\left(z_{1}, z_{2}\right) \in \mathscr{S}$. Expanding the displacement in the adherent, $\mathbf{u}^{\varepsilon}$, in Taylor series along the $x_{3}$-direction and taking into account the asymptotic expansion (7a), it results:

$$
\begin{aligned}
\mathbf{u}^{\varepsilon}\left(\mathbf{x}_{\alpha}, \pm \frac{\varepsilon}{2}\right) & =\mathbf{u}^{\varepsilon}\left(\mathbf{x}_{\alpha}, 0^{ \pm}\right) \pm \frac{\varepsilon}{2} \mathbf{u}_{, 3}^{\varepsilon}\left(\mathbf{x}_{\alpha}, 0^{ \pm}\right)+\cdots \\
& =\mathbf{u}^{0}\left(\mathbf{x}_{\alpha}, 0^{ \pm}\right)+\varepsilon \mathbf{u}^{1}\left(\mathbf{x}_{\alpha}, 0^{ \pm}\right) \pm \frac{\varepsilon}{2} \mathbf{u}_{, 3}^{0}\left(\mathbf{x}_{\alpha}, 0^{ \pm}\right)+\cdots
\end{aligned}
$$

Substituting Eqs. (7b) and (7c) together with formula (27) into continuity condition (26), it holds true:

$$
\begin{aligned}
\mathbf{u}^{0}\left(\mathbf{x}_{\alpha}, 0^{ \pm}\right)+\varepsilon \mathbf{u}^{1}\left(\mathbf{x}_{\alpha}, 0^{ \pm}\right) \pm \frac{\varepsilon}{2} \mathbf{u}_{, 3}^{0}\left(\mathbf{x}_{\alpha}, 0^{ \pm}\right)+\cdots & =\hat{\mathbf{u}}^{0}\left(\mathbf{z}_{\alpha}, \pm \frac{1}{2}\right)+\varepsilon \hat{\mathbf{u}}^{1}\left(\mathbf{z}_{\alpha}, \pm \frac{1}{2}\right)+\cdots \\
& =\overline{\mathbf{u}}^{0}\left(\mathbf{z}_{\alpha}, \pm \frac{1}{2}\right)+\varepsilon \overline{\mathbf{u}}^{1}\left(\mathbf{z}_{\alpha}, \pm \frac{1}{2}\right)+\cdots
\end{aligned}
$$

After identifying the terms in the same powers of $\varepsilon$, Eq. (28) gives:

$$
\begin{aligned}
\mathbf{u}^{0}\left(\mathbf{x}_{\alpha}, 0^{ \pm}\right) & =\hat{\mathbf{u}}^{0}\left(\mathbf{z}_{\alpha}, \pm \frac{1}{2}\right)=\overline{\mathbf{u}}^{0}\left(\mathbf{z}_{\alpha}, \pm \frac{1}{2}\right) \\
\mathbf{u}^{1}\left(\mathbf{x}_{\alpha}, 0^{ \pm}\right) \pm \frac{1}{2} \mathbf{u}_{, 3}^{0}\left(\mathbf{x}_{\alpha}, 0^{ \pm}\right) & =\hat{\mathbf{u}}^{1}\left(\mathbf{z}_{\alpha}, \pm \frac{1}{2}\right)=\overline{\mathbf{u}}^{1}\left(\mathbf{z}_{\alpha}, \pm \frac{1}{2}\right)
\end{aligned}
$$

Following a similar analysis for the stress vector, analogous results are obtained $[38,53]$ :

$$
\begin{gathered}
\sigma_{i 3}^{0}\left(\mathbf{x}_{\alpha}, 0^{ \pm}\right)=\hat{\sigma}_{i 3}^{0}\left(\mathbf{z}_{\alpha}, \pm \frac{1}{2}\right)=\bar{\sigma}_{i 3}^{0}\left(\mathbf{z}_{\alpha}, \pm \frac{1}{2}\right) \\
\sigma_{i 3}^{1}\left(\mathbf{x}_{\alpha}, 0^{ \pm}\right) \pm \frac{1}{2} \sigma_{i 3,3}^{0}\left(\mathbf{x}_{\alpha}, 0^{ \pm}\right)=\hat{\sigma}_{i 3}^{1}\left(\mathbf{z}_{\alpha}, \pm \frac{1}{2}\right)=\bar{\sigma}_{i 3}^{1}\left(\mathbf{z}_{\alpha}, \pm \frac{1}{2}\right)
\end{gathered}
$$

for $i=1,2,3$. form:

Using the above results, it is possible to rewrite Eqs. (21) and (22) in the following

$$
\begin{aligned}
& {\left[\left[\sigma_{i 3}^{0}\right]\right]=0, \quad i=1,2,3} \\
& {\left[\left[\sigma_{33}^{1}\right]\right]=-\sigma_{13,1}^{0}-\sigma_{23,2}^{0}-\left\langle\left\langle\sigma_{33,3}^{0}\right\rangle\right\rangle}
\end{aligned}
$$

where $[[f]]:=f\left(\mathbf{x}_{\alpha}, 0^{+}\right)-f\left(\mathbf{x}_{\alpha}, 0^{-}\right)$is taken to denote the jump across the surface $\mathscr{S}$ of a generic function $f$ defined on the limit configuration obtained as $\varepsilon \rightarrow 0$, as schematically outlined in Fig. 1, while it is set $\langle\langle f\rangle\rangle:=\frac{1}{2}\left(f\left(\mathbf{x}_{\alpha}, 0^{+}\right)+f\left(\mathbf{x}_{\alpha}, 0^{-}\right)\right)$.

It is worth to point out that all the equations written so far are independent of the constitutive behavior of the material. 


\subsubsection{Concerning Constitutive Equations}

The specific constitutive behavior of the materials is now introduced [38, 53]. In particular, the linearly elastic constitutive laws for the adherents and the interphase, relating the stress with the strain, are given by the equations:

$$
\begin{aligned}
\overline{\boldsymbol{\sigma}}^{\varepsilon} & =\mathbb{A}_{ \pm}\left(\mathbf{e}\left(\overline{\mathbf{u}}^{\varepsilon}\right)\right) \\
\hat{\boldsymbol{\sigma}}^{\varepsilon} & =\mathbb{B}^{\varepsilon}\left(\mathbf{e}\left(\hat{\mathbf{u}}^{\varepsilon}\right)\right)
\end{aligned}
$$

where $A_{i j k l}^{ \pm}, B_{i j k l}^{\varepsilon}$ are the elasticity tensor of the adherents and of the interphase, respectively.

It is worth pointing out that in order to achieve the interface law via this asymptotic approach, the only assumption on the constitutive behavior of constituents, to do necessarily, is that of linear elastic materials. Thereby, no assumption is herein made on the anisotropy of both constituents and on their soundness.

In what follows, within the framework of the imperfect interface approach it has been shown that it is possible to account for different interphase anisotropy conditions and for damage phenomena in the interphase.

In the following section, reference is made to the analysis of interphase behavior, detailing both the soft and hard interphase cases.

\subsubsection{Internal/Interphase Analysis}

Recalling the seven-regimes distinguish made by Benveniste and Miloh [5] (see Sect. 1), basically two of these typologies of interphase are considered in the present work. The first interphase type, called soft interphase, is defined as an interphase material whose elastic properties are linearly rescaled with respect to the interphase thickness $\varepsilon$. The second type, referred as hard interphase, is characterized by elastic moduli, which, on the contrary, do not depend on the thickness $\varepsilon$. It is worth pointing out that these hypothesis are referred to the stiffness or the softness of the interphase with respect to the neighboring media (adherents) and it does not depend on the constitutive assumptions (in terms of anisotropy) made on the interphase material. Moreover, the soft interphase behavior is, generally, the simplest constitutive hypothesis made to describe an adhesive layer (e.g. glue). Nevertheless, such an assumption can be an useful strategy in order to take into account for contact zone or thin zones between solids in which interacting phenomena occur.

The soft interface definition, as above explained, concerns the capacity to have a non-negligible displacement jump [[u]] through a surface between two bodies in contact [5, 26, 41], this kind of interface has been also referred as spring-type model. The hard interface definition, instead, concerns the capacity to have non-negligible displacement jump $[[\mathbf{u}]]$ and stress jump $[[\boldsymbol{\sigma}]]$ through a surface between two bodies in contact.

The matched asymptotic expansion method applied to soft and hard interphases gives rise to soft and hard interface laws, respectively. 
These two cases are relevant for the development of the interface laws classically used in technical problems. Moreover, models of perfect and imperfect interfaces, which are currently used in finite element codes, are known to arise from the hard and the soft interface conditions expanded at the first (zero) order [4, 5, 10, 36, 38]. The interface laws at the higher order, both in the soft and in the hard cases, are object of recent studies [53] which are recalled in the following.

\section{Soft Interphase Analysis:}

Assuming that the interphase is soft, let the interphase elasticity tensor $\mathbb{B}^{\varepsilon}$ be defined as [53]:

$$
\mathbb{B}^{\varepsilon}=\varepsilon \mathbb{B}
$$

where tensor $\mathbb{B}$ does not depend on $\varepsilon$. Referring to Voigt notation rule, its components can be expressed as:

$$
K_{k i}^{j l}:=B_{i j k l}
$$

Taking into account relations (9), (16b) and (35), the stress-strain law (34b) takes the following form:

$$
\hat{\boldsymbol{\sigma}}^{0}+\varepsilon \hat{\boldsymbol{\sigma}}^{1}=\mathbb{B}\left(\hat{\mathbf{e}}^{-1}+\varepsilon \hat{\mathbf{e}}^{0}\right)+o(\varepsilon)
$$

As Eq. (37) is true for any value of $\varepsilon$, the following expressions are derived:

$$
\begin{aligned}
& \hat{\boldsymbol{\sigma}}^{0}=\mathbb{B}\left(\hat{\mathbf{e}}^{-1}\right) \\
& \hat{\boldsymbol{\sigma}}^{1}=\mathbb{B}\left(\hat{\mathbf{e}}^{0}\right)
\end{aligned}
$$

Substituting Eq. (36) into Eq. (38a) it results:

$$
\hat{\sigma}_{i j}^{0}=B_{i j k l} \hat{e}_{k l}^{-1}=K_{k i}^{j l} \hat{e}_{k l}^{-1}
$$

and using Eq. (10), it follows that:

$$
\hat{\boldsymbol{\sigma}}^{0} \mathbf{i}_{j}=\mathbf{K}^{3 j} \hat{\mathbf{u}}_{, 3}^{0}
$$

for $j=1,2$, 3. Integrating Eq. (40) written for $j=3$, with respect to $z_{3}$, it results:

$$
\hat{\boldsymbol{\sigma}}^{0} \mathbf{i}_{3}=\mathbf{K}^{33}\left[\hat{\mathbf{u}}^{0}\right]
$$

which represents the classical law for a soft interface at the zero-order.

Recalling a recent study by Rizzoni et al. [53], it is possible to formulate the soft interface law at the one-order. Accordingly, by substituting the expression (36) into (38b) and by using formula (11) written for $k=0$, one has: 


$$
\hat{\boldsymbol{\sigma}}^{1} \mathbf{i}_{j}=\mathbf{K}^{1 j} \hat{\mathbf{u}}_{, 1}^{0}+\mathbf{K}^{2 j} \hat{\mathbf{u}}_{, 2}^{0}+\mathbf{K}^{3 j} \hat{\mathbf{u}}_{, 3}^{1}
$$

for $j=1,2,3$. Moreover, by taking into account formula (40), written for $j=1,2$, the equilibrium Eq. (20) explicitly becomes:

$$
\left(\mathbf{K}^{31} \hat{\mathbf{u}}_{, 3}^{0}\right)_{, 1}+\left(\mathbf{K}^{32} \hat{\mathbf{u}}_{, 3}^{0}\right)_{, 2}+\left(\hat{\boldsymbol{\sigma}}^{1} \mathbf{i}_{3}\right)_{, 3}=\mathbf{0}
$$

and thus, integrating with respect to $z_{3}$ between $-\frac{1}{2}$ and $\frac{1}{2}$, it gives:

$$
\left[\hat{\boldsymbol{\sigma}}^{1} \mathbf{i}_{3}\right]=-\mathbf{K}^{31}\left[\hat{\mathbf{u}}^{0}\right]_{, 1}-\mathbf{K}^{32}\left[\hat{\mathbf{u}}^{0}\right]_{, 2}
$$

It is worth remarking that the stress components $\hat{\sigma}_{i 3}^{0}$ (with $i=1,2,3$ ) are independent of $z_{3}$, because of the Eq. (19). Consequently, taking into account Eq. (40) written for $j=3$, the derivatives $\hat{u}_{i, 3}^{0}$ are also independent of $z_{3}$; thus, the displacement components $\hat{u}_{i}^{0}$ are a linear functions of $z_{3}$. Therefore, Eq. (44) reveals that the stress components $\hat{\sigma}_{i 3}{ }^{1}$, with $i=1,2,3$, are linear functions in $z_{3}$, allowing to write the following representation form for the stress components:

$$
\hat{\boldsymbol{\sigma}}^{1} \mathbf{i}_{3}=\left[\hat{\boldsymbol{\sigma}}^{1} \mathbf{i}_{3}\right] z_{3}+\left\langle\hat{\boldsymbol{\sigma}}^{1} \mathbf{i}_{3}\right\rangle
$$

where $\langle f\rangle\left(\mathbf{z}_{\alpha}\right):=\frac{1}{2}\left(f\left(\mathbf{z}_{\alpha}, \frac{1}{2}\right)+f\left(\mathbf{z}_{\alpha},-\frac{1}{2}\right)\right)$. Substituting Eq. (42) written for $j=3$ into expression (45) and integrating with respect to $z_{3}$ it yields:

$$
\left\langle\hat{\boldsymbol{\sigma}}^{1} \mathbf{i}_{3}\right\rangle=\mathbf{K}^{\alpha 3}\left\langle\hat{\mathbf{u}}^{0}\right\rangle_{, \alpha}+\mathbf{K}^{33}\left[\hat{\mathbf{u}}^{1}\right]
$$

where the sum over $\alpha=1,2$ is implied. Combining Eqs. (44)-(46), it results:

$$
\begin{aligned}
\hat{\boldsymbol{\sigma}}^{1}\left(\mathbf{z}_{\alpha}, \pm \frac{1}{2}\right) \mathbf{i}_{3}= & \mathbf{K}^{33}\left[\hat{\mathbf{u}}^{1}\right]\left(\mathbf{z}_{\alpha}\right)+\frac{1}{2}\left(\mathbf{K}^{\alpha 3} \mp \mathbf{K}^{3 \alpha}\right) \hat{\mathbf{u}}_{, \alpha}^{0}\left(\mathbf{z}_{\alpha}, \frac{1}{2}\right) \\
& +\frac{1}{2}\left(\mathbf{K}^{\alpha 3} \pm \mathbf{K}^{3 \alpha}\right) \hat{\mathbf{u}}_{, \alpha}^{0}\left(\mathbf{z}_{\alpha},-\frac{1}{2}\right)
\end{aligned}
$$

The soft interface laws at zero-order and at one-order, expressed by Eqs. (41) and (47) respectively, have to be formulated in their final form in terms of the stresses and displacements fields in the final configuration (see Fig. 1c). To this aim, using the matching relations (29)-(32), the final formulations of the soft interface laws at zero-order and at one-order, respectively, are the following [53]:

$$
\begin{aligned}
\boldsymbol{\sigma}^{0}(\cdot, 0) \mathbf{i}_{3}= & \mathbf{K}^{33}\left[\left[\mathbf{u}^{0}\right]\right], \\
\boldsymbol{\sigma}^{1}\left(\cdot, 0^{ \pm}\right) \mathbf{i}_{3}= & \mathbf{K}^{33}\left(\left[\left[\mathbf{u}^{1}\right]\right]+\left\langle\left\langle\mathbf{u}_{, 3}^{0}\right\rangle\right\rangle\right)+\frac{1}{2}\left(\mathbf{K}^{\alpha 3} \mp \mathbf{K}^{3 \alpha}\right) \mathbf{u}_{, \alpha}^{0}\left(\cdot, 0^{+}\right) \\
& +\frac{1}{2}\left(\mathbf{K}^{\alpha 3} \pm \mathbf{K}^{3 \alpha}\right) \mathbf{u}_{, \alpha}^{0}\left(\cdot, 0^{-}\right) \mp \frac{1}{2} \sigma_{, 3}^{0}\left(\cdot, 0^{ \pm}\right) \mathbf{i}_{3}
\end{aligned}
$$


where the symbol $(\cdot)$ represents the coordinates $\left(x_{1}, x_{2}\right)$ in a generic point of the system $\Omega_{+} \cup \Omega_{-}$in the final configuration. In detail, Eq. (48) represents the classical spring-type interface law, derived from an interphase characterized by a finite stiffness. Moreover, Eq. (49) allows to evaluate the stress vector at the higher (one) order, highlighting that the stress vector $\sigma^{1}\left(\cdot, 0^{ \pm}\right) \mathbf{i}_{3}$ depends not only on displacement jump at one-order but also on the displacement and stress fields evaluated at the zero-order and their derivatives.

In order to have a complete expression of the effective stress field in the reference configuration (see Fig. 1a), Eqs. (16b) and (7c) must be substituted in Eqs. (48) and (49). Finally, it results:

$$
\begin{aligned}
\boldsymbol{\sigma}^{\varepsilon}\left(\cdot, 0^{ \pm}\right) \mathbf{i}_{3} \approx & \mathbf{K}^{33}\left[\left[\mathbf{u}^{\varepsilon}\right]\right]+\varepsilon\left(\mathbf{K}^{33}\left\langle\left\langle\mathbf{u}_{, 3}^{\varepsilon}\right\rangle\right\rangle\right. \\
& +\frac{1}{2}\left(\mathbf{K}^{\alpha 3} \mp \mathbf{K}^{3 \alpha}\right) \mathbf{u}_{, \alpha}^{\varepsilon}\left(\cdot, 0^{+}\right) \\
& \left.+\frac{1}{2}\left(\mathbf{K}^{\alpha 3} \pm \mathbf{K}^{3 \alpha}\right) \mathbf{u}_{, \alpha}^{\varepsilon}\left(\cdot, 0^{-}\right) \mp \frac{1}{2} \boldsymbol{\sigma}_{, 3}^{\varepsilon}\left(\cdot, 0^{ \pm}\right) \mathbf{i}_{3}\right)
\end{aligned}
$$

It is worth remarking that Eq. (50) improves the classic interface law at zero-order by linearly linking the stress vector and the relative displacement via a higher order term, involving the in-plane first derivatives of the displacement. Moreover, (50) allows to clearly quantify the error committed in the interface constitutive equation by modeling a $\varepsilon$-thick layer with a soft interface law at the zero-order (first right-side term in Eq. (50)). In particular, if the in-plane gradient of displacement and/or the out-of-plane gradient of stress are relevant, they can be neglected in the interface constitutive law.

\section{Hard Interphase Analysis:}

For a hard interphase, the elasticity tensor $\mathbb{B}^{\varepsilon}$ takes the following form $[14,38,53]$ :

$$
\mathbb{B}^{\varepsilon}=\mathbb{B}
$$

where the tensor $\mathbb{B}$ does not depend on $\varepsilon$, and $\mathbf{K}^{j l}$ is still taken to denote the matrices such that $K_{k i}^{j l}:=B_{i j k l}$ (Voigt notation).

Taking into account relations (9) and (16b), the stress-strain Eq. (34b) takes the following form:

$$
\hat{\boldsymbol{\sigma}}^{0}+\varepsilon \hat{\boldsymbol{\sigma}}^{1}=\mathbb{B}\left(\varepsilon^{-1} \hat{\mathbf{e}}^{-1}+\hat{\mathbf{e}}^{0}+\varepsilon \hat{\mathbf{e}}^{1}\right)+o(\varepsilon)
$$


As Eq. (52) is true for any value of $\varepsilon$, the following conditions are derived:

$$
\begin{aligned}
\mathbf{0} & =\mathbb{B}\left(\hat{\mathbf{e}}^{-1}\right) \\
\hat{\boldsymbol{\sigma}}^{0} & =\mathbb{B}\left(\hat{\mathbf{e}}^{0}\right)
\end{aligned}
$$

Taking into account Eq. (10) and the positive definiteness of the tensor $\mathbb{B}$, relation (53a) gives:

$$
\hat{\mathbf{u}}_{, 3}^{0}=0 \Rightarrow\left[\hat{\mathbf{u}}^{0}\right]=\mathbf{0}
$$

which corresponds to the kinematics of the perfect interface.

Substituting Eq. (11) written for $k=0$ into (53b) one has:

$$
\hat{\boldsymbol{\sigma}}^{0} \mathbf{i}_{j}=\mathbf{K}^{1 j} \hat{\mathbf{u}}_{, 1}^{0}+\mathbf{K}^{2 j} \hat{\mathbf{u}}_{, 2}^{0}+\mathbf{K}^{3 j} \hat{\mathbf{u}}_{, 3}^{1}
$$

for $j=1,2$, 3. Integrating Eq. (55) written for $j=3$, with respect to $z_{3}$, it results:

$$
\left[\hat{\mathbf{u}}^{1}\right]=\left(\mathbf{K}^{33}\right)^{-1}\left(\hat{\boldsymbol{\sigma}}^{0} \mathbf{i}_{3}-\mathbf{K}^{\alpha 3} \hat{\mathbf{u}}_{, \alpha}^{0}\right)
$$

Recalling the Eq. (55) (written for $j=1,2$ ), equilibrium equation Eq. (20) explicitly becomes:

$$
\left(\mathbf{K}^{11} \hat{\mathbf{u}}_{, 1}^{0}+\mathbf{K}^{21} \hat{\mathbf{u}}_{, 2}^{0}+\mathbf{K}^{31} \hat{\mathbf{u}}_{, 3}^{1}\right)_{, 1}+\left(\mathbf{K}^{12} \hat{\mathbf{u}}_{, 1}^{0}+\mathbf{K}^{22} \hat{\mathbf{u}}_{, 2}^{0}+\mathbf{K}^{32} \hat{\mathbf{u}}_{, 3}^{1}\right)_{, 2}+\left(\hat{\boldsymbol{\sigma}}^{1} \mathbf{i}_{3}\right)_{, 3}=\mathbf{0}
$$

and thus, integrating with respect to $z_{3}$ between $-1 / 2$ and $1 / 2$ and using (56), it gives:

$$
\begin{aligned}
{\left[\hat{\boldsymbol{\sigma}}^{1} \mathbf{i}_{3}\right] } & =\left(-\mathbf{K}^{\alpha \beta} \hat{\mathbf{u}}_{, \beta}^{0}-\mathbf{K}^{3 \alpha}\left[\hat{\mathbf{u}}^{1}\right]\right)_{, \alpha} \\
& =\left(-\mathbf{K}^{\alpha \beta} \hat{\mathbf{u}}_{, \beta}^{0}-\mathbf{K}^{3 \alpha}\left(\mathbf{K}^{33}\right)^{-1}\left(\hat{\boldsymbol{\sigma}}^{0} \mathbf{i}_{3}-\mathbf{K}^{\beta 3} \hat{\mathbf{u}}_{, \beta}^{0}\right)\right)_{, \alpha}
\end{aligned}
$$

with the Greek indexes $(\alpha, \beta=1,2)$ are related, as usual, to the in-plane $\left(x_{1}, x_{2}\right)$ quantities.

It is worth noting that in Eq. (58) higher order effects occur and they are related to the appearance of in-plane derivatives, which are usually neglected in the classical first (zero) order theories of interfaces [14, 38, 53]. These new terms are related to second-order derivatives and as a consequence, indirectly, to the curvature of the deformed interface. By non-neglecting these terms it is possible to model a membrane effect in the adhesive [53].

In the hard case also, it is possible to derive a final form of the interface laws in terms of the stresses and displacements fields in the final configuration (Fig. 1c). Using matching relations (29)-(32) the interface laws, calculated both at zero-order and at one-order, can be rewritten as follows [14, 53]: 


$$
\begin{aligned}
& {\left[\left[\mathbf{u}^{0}\right]\right]=\mathbf{0}} \\
& {\left[\left[\mathbf{u}^{1}\right]\right]=-\left(\mathbf{K}^{33}\right)^{-1}\left(\boldsymbol{\sigma}^{0} \mathbf{i}_{3}-\mathbf{K}^{\alpha 3} \mathbf{u}_{, \alpha}^{0}\right)-\left\langle\left\langle\mathbf{u}_{, 3}^{0}\right\rangle\right\rangle} \\
& {\left[\left[\boldsymbol{\sigma}^{0} \mathbf{i}_{3}\right]\right]=\mathbf{0}} \\
& {\left[\left[\boldsymbol{\sigma}^{1} \mathbf{i}_{3}\right]\right]=\left(-\mathbf{K}^{\alpha \beta} \mathbf{u}_{, \beta}^{0}+\mathbf{K}^{3 \alpha}\left(\mathbf{K}^{33}\right)^{-1}\left(\boldsymbol{\sigma}^{0} \mathbf{i}_{3}-\mathbf{K}^{\beta 3} \mathbf{u}_{, \beta}^{0}\right)\right)_{, \alpha}-\left\langle\left\langle\boldsymbol{\sigma}_{, 3}^{0} \mathbf{i}_{3}\right\rangle\right\rangle}
\end{aligned}
$$

Equations (59) and (61) represent the classical perfect interface law characterized by the continuity of the displacement and stress vector fields [5]. Additionally, Eqs. (60) and (62) are imperfect interface conditions, allowing jumps in the displacement and in the stress vector fields at the higher (one) order across the interface $\mathscr{S}$ [53]. Moreover, Eqs. (60) and (62) highlight that these jumps depend on the displacement and the stress fields at the zero-order and on their first and second order derivatives [14].

As done in the soft case, the constitutive law for the hard interface written in terms of displacement jumps and stresses in the reference configuration (Fig. 1a) can be derived (with reference to $[14,53])$. By considering the expansions (16a) and (7a) combined with Eqs. (59)-(62). The obtained imperfect interface laws reads as:

$$
\begin{aligned}
{\left[\left[\mathbf{u}^{\varepsilon}\right]\right] \approx } & -\varepsilon\left(\left(\mathbf{K}^{33}\right)^{-1}\left(\boldsymbol{\sigma}^{\varepsilon} \mathbf{i}_{3}+\mathbf{K}^{\alpha 3} \mathbf{u}_{, \alpha}^{\varepsilon}\right)-\left\langle\left\langle\mathbf{u}_{, 3}^{\varepsilon}\right\rangle\right\rangle\right) \\
{\left[\left[\boldsymbol{\sigma}^{\varepsilon} \mathbf{i}_{3}\right]\right] \approx } & \varepsilon\left(\left(-\mathbf{K}^{\alpha \beta} \mathbf{u}_{, \beta}^{\varepsilon}+\mathbf{K}^{3 \alpha}\left(\mathbf{K}^{33}\right)^{-1}\left(\boldsymbol{\sigma}^{\varepsilon} \mathbf{i}_{3}-\mathbf{K}^{\beta 3} \mathbf{u}_{, \beta}^{\varepsilon}\right)\right)_{, \alpha}\right. \\
& \left.-\left\langle\left\langle\boldsymbol{\sigma}_{, 3}^{\varepsilon} \mathbf{i}_{3}\right\rangle\right\rangle\right)
\end{aligned}
$$

\subsection{Homogenization in Non-interacting Approximation (NIA) for Microcracked Media}

The class of inhomogeneities considered in the paper is that of planar microcracks, both in the two-dimensional framework (rectilinear cracks) and in the threedimensional framework (penny-shaped cracks). The considered imperfect interphase $\mathscr{B}^{\varepsilon}$, defined as the thin layer having $\mathscr{S}$ as the middle section and $\varepsilon$ as the uniform small thickness, is weakened by non-interacting penny-shaped microcracks of radius $b$. Cracks are assumed to be characterized by a periodic transversally isotropic distribution with symmetry axis $\mathbf{i}_{3}$. Moreover, the non-interacting approximation (NIA) is enforced [57], accordingly, each crack does not experience mechanical interaction by surrounding cracks. Within the NIA framework, one recalls that the microcracks contribution to the material effective properties is obtained as a summation over the contribution of a single crack (or a family of cracks with characteristic length and orientation [34]). As a result, a $\varepsilon$-thick representative elementary volume (REV) of the interphase comprising a single crack can be conveniently introduced as sketched in Fig. 2. Note that in the case of a family of parallel cracks (with same orientation), it is possible to identify this family by an equivalent crack with average radius. 
Fig. $2 R E V$ with a crack-sketch of the $\varepsilon$-thick representative elementary volume (REV) taken into account in the 3-d homogenization process

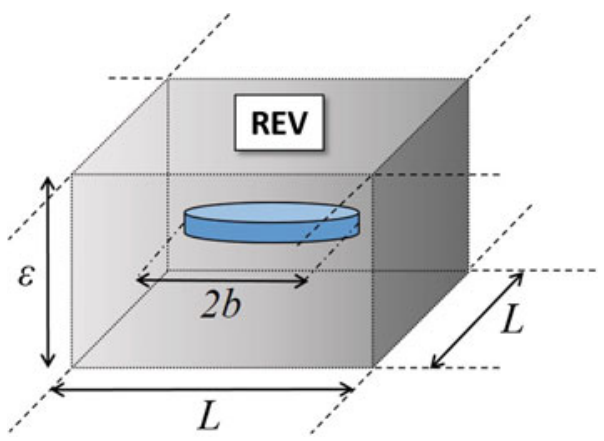

The non-interacting approximation is particularly useful for cracked materials, basically, for two reasons:

- it appears to be relatively accurate to high crack density, where local interaction effects become substantial, this evidence can be due to the fact that presence of cracks does not change the average stress in the matrix;

- substantial progress has been made in analyzing shape factors for cracks of complex shapes.

It is worth remarking that, in the following text, for the sake of briefness, the word crack is often used instead of microcracks; however the whole formulation that will be discussed belongs to a micromechanics framework.

Mathematically, a crack is characterized by a surface of discontinuity experienced by displacements (or temperature) when external fields are applied. Property contribution tensors have rather specific structure for cracks. A complicating factor is that cracks often have irregular shapes (including non-planar and intersected configurations). Nevertheless, this shortcoming is not taken into account in the present paper.

NIA formulation have two dual forms, which in the following will be referred as stress-based approach and strain-based approach. They correspond to obtain the property contribution tensor via a summation of compliance or stiffness contributions of individual inhomogeneities, respectively. In the following sections, the general formulation of these homogenization approaches for microcracked media in the NIA framework is outlined.

\subsubsection{Stress-Based Approach}

Generally, the additional strain tensor (averaged over the domain $\Omega$ of volume $V$ ) due to the presence of a pore is given by the following integral over the pore boundary $\partial \Omega_{p}$ :

$$
\Delta \varepsilon=-\frac{1}{V} \int_{\partial \Omega_{p}}\left(\mathbf{u} \otimes^{s} \mathbf{n}\right) \mathrm{d} S
$$


where $\otimes^{s}$ is the symmetric tensorial product, $\mathbf{u}$ is the displacement vector, $\mathbf{n}$ is a unit normal to $\partial \Omega_{p}$ directed inward the pore.

Let $v^{+}$and $v^{-}$be the displacements at the crack boundaries $\Gamma^{+}$and $\Gamma^{-}$with $\Gamma=$ $\Gamma^{+} \cup \Gamma^{-}$. Denote also as $\mathbf{u}_{\text {cod }}=\left\langle\boldsymbol{v}^{+}-\boldsymbol{v}^{-}\right\rangle=\left[\int_{\Gamma}\left(\boldsymbol{v}^{+}-\boldsymbol{v}^{-}\right) \mathrm{d} \Gamma\right] /|\Gamma|$ the average measure of the displacement jump across the crack, in the following referred to as crack opening displacement (COD) vector. Where $|\Gamma|$ is the measure of the crack surface. In this case, Eq. (65) takes the form:

$$
\Delta \varepsilon=\frac{1}{V} \int_{\Gamma^{+}}\left([\boldsymbol{v}] \otimes^{s} \mathbf{n}\right) \mathrm{d} S
$$

where $\mathbf{n}$ is the normal unit vector of the crack surface and $[\boldsymbol{v}]=\left(\boldsymbol{v}^{+}-\boldsymbol{v}^{-}\right)$is the displacement discontinuity vector along $\Gamma$. Calculation of the integral in terms of remotely applied stress $\sigma^{0} \equiv \sigma$ would yield the $\mathbb{H}$-tensor of the crack, defined as:

$$
\Delta \boldsymbol{\varepsilon}=\frac{V^{p}}{V}\left(\mathbb{H}: \boldsymbol{\sigma}^{0}\right)
$$

For a flat (planar) crack ( $\mathbf{n}$ is constant along $\Gamma$ ), the additional strain $\Delta \varepsilon$ becomes:

$$
\Delta \varepsilon=\frac{1}{V}\left(\mathbf{u}_{c o d} \otimes{ }^{s} \mathbf{n}\right) \Gamma
$$

Equations (66) and (68) are an immediate consequence of a footnote remark in the famous work by Hill [31].

Let recall that under the approximation of non-interacting cracks, each crack is embedded into the $\boldsymbol{\sigma}$-field and it does not experience any influence of other cracks. As a result, for a flat crack of any shape, a second-rank crack compliance tensor $\mathbf{B}$ can be introduced that relates vector $\mathbf{u}_{\text {cod }}$ to the vector of uniform traction $\mathbf{T}_{n}=\boldsymbol{\sigma} \cdot \mathbf{n}$ induced at the crack site by the far-field $\sigma[34,35,43,60]$ :

$$
\mathbf{u}_{c o d}=\mathbf{T}_{n}^{T} \cdot \mathbf{B}
$$

Therefore, according to the hypothesis of linear elasticity of materials and absence of friction along crack faces, the average COD vector for each crack is expressed in terms of the vector of uniform traction $\mathbf{T}_{n}$.

Since $\mathbf{B}$ is a symmetric tensor (as follows from application of the Betti reciprocity theorem to the normal and shear tractions on a crack), three orthogonal principal directions of the crack compliance exist: application of a uniform traction in one of them does not generate components of vector $\mathbf{u}_{\text {cod }}$ in the other two directions. If the matrix is isotropic, $\mathbf{n}$ is one of them and the other two, $\mathbf{t}$ and $\mathbf{s}$, lie in the crack plane, as follows:

$$
\mathbf{B}=B_{n n}(\mathbf{n} \otimes \mathbf{n})+B_{t t}(\mathbf{t} \otimes \mathbf{t})+B_{s s}(\mathbf{s} \otimes \mathbf{s})
$$


As a definition, let introduce the average, over in-plane directions $\boldsymbol{\tau}$, shear crack compliance that is of importance for the effective elastic properties of a solid with multiple cracks [57]:

$$
B_{T}=\frac{\left(B_{t t}+B_{s s}\right)}{2}
$$

It is worth to remark that the $\mathbf{B}$ tensor has to be specialized with respect to the bulk material properties.

Within the NIA framework, the problem of quantitative characterization of microstructures is reduced to find the proper microstructural parameter of inhomogeneities in whose terms the effective property of interest, compliance tensor has to be expressed [35]. Generally, the concentration parameters of inhomogeneities in the context of the elastic properties are better identified via the structure of the additional elastic potential $\Delta f$.

For flat cracks, recalling Eshelby's theory [16], the elastic potential $f(\sigma)$ of the effective microcracked material, written in terms of microstructural quantities defined on the crack surfaces $\Gamma^{i}$, is [34, 57, 59]:

$$
f(\boldsymbol{\sigma})=f_{0}(\boldsymbol{\sigma})+\Delta f=\frac{1}{2} \boldsymbol{\sigma}: \mathbb{S}_{0}: \boldsymbol{\sigma}+\frac{1}{2 V} \sum_{i}\left(\mathbf{T}_{n}^{T} \cdot \mathbf{u}_{c o d}\right)^{i} \Gamma^{i}
$$

where $f_{0}(\boldsymbol{\sigma})$ is, as usually, the potential of the bulk matrix (interphase) and the perturbation term $\Delta f$ is obtained as a sum of the contributions of individual cracks, i.e. $\sum_{i}$ is a summation over the families of microcracks of length $2 l^{i}$ and normal vector $\mathbf{n}^{i}$. Recall that the tensor $\mathbb{S}_{0}$ appearing in Eq. (72) is the compliance tensor of the virgin interphase.

In the important case of randomly oriented circular cracks (penny-shaped) of radii $b^{i}$, their concentration is characterized by the crack density parameter introduced by Bristow [7]:

$$
\rho=\frac{1}{V} \sum_{i} b^{(i) 3}
$$

that in the two-dimensional case of randomly oriented rectilinear cracks of meanlength $l^{i}$ becomes:

$$
\rho=\frac{1}{A} \sum_{i} l^{(i) 2}
$$

This parameter was generalized by Budiansky and O'Connell [8] to the elliptical in-plane shapes, of areas $S^{(i)}$ and perimeters $P^{(i)}$ (provided aspect ratios of ellipses are identical) as:

$$
\rho=\frac{2}{\pi} \frac{1}{V} \sum_{i}\left(\frac{S^{2}}{P}\right)^{i}
$$

For non-random crack orientations, the crack density tensor was introduced by Kachanov [34]: 


$$
\boldsymbol{\alpha}=\frac{1}{V} \sum_{i}\left(b^{3} \mathbf{n} \otimes \mathbf{n}\right)^{i} \quad\left(\boldsymbol{\alpha}=\frac{1}{A} \sum_{i}\left(l^{2} \mathbf{n} \otimes \mathbf{n}\right)^{i} \text { in 2-D case }\right)
$$

with $\rho=\operatorname{Tr} \boldsymbol{\alpha}$. Kachanov introduced also a fourth-rank density tensor in threedimensional case

$$
\frac{1}{V} \sum_{i}\left(b^{3} \mathbf{n} \otimes \mathbf{n} \otimes \mathbf{n} \otimes \mathbf{n}\right)^{i}
$$

which in general causes a small deviation from orthotropy.

As an example, in the three-dimensional case of an isotropic material weakened by open penny-shaped cracks the elastic potential $f$ is [35]:

$$
f=f_{0}+\frac{8\left(1-v_{0}^{2}\right)}{3\left(1-\frac{\nu_{0}}{2}\right) E_{0}}\left[(\boldsymbol{\sigma} * \boldsymbol{\sigma}): \boldsymbol{\alpha}-\frac{\nu_{0}}{2}\left(\boldsymbol{\sigma}: \frac{1}{V} \sum_{i}\left(b^{3} \mathbf{n} \otimes \mathbf{n} \otimes \mathbf{n} \otimes \mathbf{n}\right)^{i}: \boldsymbol{\sigma}\right)\right]
$$

\subsubsection{Strain-Based Approach}

Goidescu-type formulation is developed within the framework of 2-D homogenization problems [20, 21]. It extends the micromechanical approach proposed by Andrieux et al. [2] and leads to a closed-form expression of the macroscopic free energy of a 2D orthotropic elastic medium weakened by arbitrarily oriented microcracks in the dilute limit assumption. It exists a large amount of literature about the homogenization of microcracked media following a strain-based approach $[25,31-33,47]$. It is worth to recall that within the framework of this approach the stiffness contribution tensor $\Delta \mathbb{C}$ are derived starting from a free energy $\mathscr{W}$. As done above for the stress-based approach, let the general background be outlined. Particularly, reference is herein made to the two-dimensional formulation by $[20,21]$.

Let consider a RVE of total area $\mathscr{A}$, the bulk matrix is assumed to be weakened by an array of $\mathscr{N}$ families of flat microcracks with arbitrary orientation relative to orthotropic axes and mean length $2 l^{i}$, which occupy the domain $\omega$. As a general recall [33], the macroscopic stress $\boldsymbol{\Sigma}$ and strain $\mathbf{E}$ tensors and the macroscopic free energy $\mathscr{W}$ on a cell $\mathscr{A}$ are respectively defined as average values of microscopic stress $\sigma$ and strain $\varepsilon$ fields and local free energy. Let denote by $\bar{A}=\mathscr{A}-\omega$ the area of the matrix phase, $\mathbf{v}(\mathbf{x})$ the outward unit normal to $\omega$ and $\mathbf{T}(\mathbf{x}, \mathbf{v}(\mathbf{x}))$ the traction along the crack faces for any point $\mathbf{x} \in \omega$. Decomposition of local fields over the RVE and application of the divergence theorem allow to relate macroscopic and microscopic quantities [31]. For the macroscopic stress $\boldsymbol{\Sigma}$, one has:

$$
\boldsymbol{\Sigma}=\langle\boldsymbol{\sigma}\rangle_{\mathscr{A}}=\langle\boldsymbol{\sigma}\rangle_{\mathscr{A}}+\frac{\mathscr{N}}{2} \int_{\omega}\left(\mathbf{T}(\mathbf{x}, \mathbf{v}(\mathbf{x})) \otimes^{s} \mathbf{x}\right) \mathrm{d} x=\langle\boldsymbol{\sigma}\rangle_{\mathscr{A}}
$$


For the macroscopic strain $\mathbf{E}$ one has, recalling Eq. (66):

$$
\mathbf{E}=\langle\boldsymbol{\varepsilon}\rangle_{\mathscr{A}}=\langle\boldsymbol{\varepsilon}\rangle_{\mathscr{A}}+\frac{\mathscr{N}}{2} \int_{\omega^{+}}\left([\boldsymbol{v}(\mathbf{x})] \otimes^{s} \mathbf{n}\right) \mathrm{d} x
$$

with the surface integral operator $\langle\bullet\rangle_{\mathscr{M}}=\frac{1}{|\mathscr{M}|} \int_{\mathscr{M}}(\bullet) \mathrm{d} S$, and $\mathbf{v}(\mathbf{x})=\mathbf{n}$ for $\mathbf{x} \in \omega$ the unit vector normal to the cracks, supposed to be constant along $\omega$ for flat and regular cracks. It is worth recalling that both Eqs. (66) and (80) are generalization of the Hill lemma for continuous media and they are directly derived from a footnote remark in his work [31]. From Eq. (80) is pointed out that the average strain field on the solid part $\langle\boldsymbol{\varepsilon}\rangle_{\mathscr{A}}$ is therefore not sufficient to describe $\mathbf{E}$, the contribution of displacements jump [v] on the cracks must be taken into account in its expression.

The macroscopic free energy of the material is a finite quantity exclusively defined on the matrix part of the material, that is:

$$
\mathscr{W}=\frac{1}{2}\left\langle\boldsymbol{\varepsilon}: \mathbb{C}_{0}: \boldsymbol{\varepsilon}\right\rangle_{\mathscr{A}}
$$

For microcracked media it has been established, among other by Telega [58], that the following equation holds:

$$
\mathscr{W}=\frac{1}{2} \int_{\partial \overline{\mathscr{A}}} \boldsymbol{v}(\mathbf{x}) \cdot \boldsymbol{\sigma}(\mathbf{x}) \cdot \mathbf{v}(\mathbf{x}) \mathrm{d} x=\frac{1}{2} \boldsymbol{\Sigma}: \mathbf{E}-\frac{\mathscr{N}}{2} \int_{\omega^{+}}[\boldsymbol{v}(\mathbf{x})] \cdot \boldsymbol{\sigma}(\mathbf{x}) \cdot \mathbf{n} \mathrm{d} x
$$

with $\partial \overline{\mathscr{A}}=\partial \mathscr{A} \cup \omega$ the boundary of the solid matrix.

Let consider an uniform boundary condition applied on the boundary $\partial \mathscr{A}$ of the RVE, given in terms of stresses as follows:

$$
\boldsymbol{\sigma}(\mathbf{x}) \cdot \mathbf{v}(\mathbf{x})=\boldsymbol{\Sigma} \cdot \mathbf{v}(\mathbf{x}) \forall \mathbf{x} \in \partial \mathscr{A}
$$

Within the framework of the strain-type approach, in order to derive the local fields involved and to determine the effective microcracks contribution, the elastic problem $\mathscr{P}$ is decomposed into two sub-problems $\mathscr{P}^{(1)}$ and $\mathscr{P}^{(2)}$ [2]:

- in the sub-problem $\mathscr{P}^{(1)}$, the displacement field $\mathbf{u}^{(1)}$ corresponds to that of the homogeneous virgin material subjected to uniform stress conditions; accordingly the related local stress $\sigma^{(1)}$ and strain $\boldsymbol{\varepsilon}^{(1)}$ fields are uniform and must comply with the average stress rule $\boldsymbol{\Sigma}=\left\langle\boldsymbol{\sigma}^{(1)}\right\rangle_{\mathscr{A}}$ and $\mathbf{E}^{(1)}=\left\langle\boldsymbol{\varepsilon}^{(1)}\right\rangle_{\mathscr{A}}=\left[\mathbb{C}_{0}\right]^{-1}: \boldsymbol{\Sigma}$

- for the sub-problem $\mathscr{P}^{(2)}$, the displacement field $\mathbf{u}^{(2)}$ is induced by the displacement jump $\left[\boldsymbol{v}\right.$ ] between the crack faces; the related local stress $\boldsymbol{\sigma}^{(2)}$ is in this case self-equilibrated, i.e. $\left\langle\boldsymbol{\sigma}^{(2)}\right\rangle_{\mathscr{A}}=0$ from (79); besides, since $\left\langle\boldsymbol{\varepsilon}^{(2)}\right\rangle_{\bar{A}}=\left[\mathbb{C}_{0}\right]^{-1}$ : $\left\langle\boldsymbol{\sigma}^{(2)}\right\rangle_{\mathscr{A}}=0$; the macroscopic strain reads from (80):

$$
\mathbf{E}^{(2)}=\frac{\mathscr{N}}{2} \int_{\omega^{+}}([\boldsymbol{v}(\mathbf{x})] \otimes \mathbf{n}+\mathbf{n} \otimes[\boldsymbol{v}(\mathbf{x})]) \mathrm{d} x
$$


Introducing two scalar variables $\beta$ and $\gamma$ related to the normal $\left[\mathbf{u}_{N}(\mathbf{x})\right]=[\boldsymbol{v}(\mathbf{x})] \cdot \mathbf{n}$ and tangential $\left[\mathbf{u}_{T}(\mathbf{x})\right]=[\boldsymbol{v}(\mathbf{x})] \cdot \mathbf{t}$ average displacement jump components on the cracks faces:

$$
\beta=\mathscr{N} \int_{\omega^{+}}\left[\mathbf{u}_{N}(\mathbf{x})\right] \mathrm{d} x \quad \gamma=\mathscr{N} \int_{\omega^{+}}\left[\mathbf{u}_{T}(\mathbf{x})\right] \mathrm{d} x
$$

the macroscopic strain in $\mathscr{P}^{(2)}$ reads as:

$$
\mathbf{E}^{(2)}=\beta \mathbf{n} \otimes \mathbf{n}+\frac{\gamma}{2}(\mathbf{n} \otimes \mathbf{t}+\mathbf{t} \otimes \mathbf{n})
$$

where $(\mathbf{n}, \mathbf{t})$ define an integral orthonormal basis for the crack.

According with the decomposition, the overall macroscopic strain is:

$$
\mathbf{E}=\mathbf{E}^{(1)}+\mathbf{E}^{(2)}
$$

Moreover, the overall free energy per unit surface $\mathscr{W}$ defined by Eq. (81) with $\varepsilon=$ $\boldsymbol{\varepsilon}\left(\mathbf{u}^{(1)}+\mathbf{u}^{(2)}\right)$ can be expressed as the sum of two terms [2]:

$$
\mathscr{W}=\frac{1}{2}\left\langle\left(\boldsymbol{\varepsilon}^{(1)}+\boldsymbol{\varepsilon}^{(2)}\right): \mathbb{C}_{0}:\left(\boldsymbol{\varepsilon}^{(1)}+\boldsymbol{\varepsilon}^{(2)}\right)\right\rangle_{\overline{\mathscr{A}}}=\mathscr{W}^{(1)}+\mathscr{W}^{(2)}
$$

for which have been taken into account the uniformity of $\boldsymbol{\varepsilon}^{(1)}$ and the property $\left\langle\boldsymbol{\varepsilon}^{(2)}\right\rangle_{\mathscr{A}}=0$.

$\mathscr{W}^{(1)}$ is the free energy of the virgin material related to the problem $\mathscr{P}^{(1)}$ :

$$
\mathscr{W}^{(1)}=\frac{1}{2}\left\langle\boldsymbol{\varepsilon}^{(1)}: \mathbb{C}_{0}: \boldsymbol{\varepsilon}^{(1)}\right\rangle_{\mathscr{A}}=\frac{1}{2} \mathbf{E}^{(1)}: \mathbb{C}_{0}: \mathbf{E}^{(1)}
$$

and the terms $\mathscr{W}^{(2)}$ is related to the contribution of the jump displacement in problem $\mathscr{P}^{(2)}$. It follows from Eq. (82) that:

$$
\begin{aligned}
\mathscr{W}^{(2)} & =\frac{1}{2}\left\langle\boldsymbol{\varepsilon}^{(2)}: \mathbb{C}_{0}: \boldsymbol{\varepsilon}^{(2)}\right\rangle_{\overline{\mathscr{A}}}=-\frac{\mathscr{N}}{2} \int_{\omega^{+}}[\boldsymbol{v}(\mathbf{x})] \cdot \boldsymbol{\sigma}^{(2)}(\mathbf{x}) \cdot \mathbf{n} \mathrm{d} x \\
& =-\frac{\mathscr{N}}{2} \int_{\omega^{+}}\left(\left[\mathbf{u}_{N}(\mathbf{x})\right] \mathbf{n} \cdot \boldsymbol{\sigma}^{(2)}(\mathbf{x}) \cdot \mathbf{n}+\left[\mathbf{u}_{T}(\mathbf{x})\right] \mathbf{n} \cdot \boldsymbol{\sigma}^{(2)}(\mathbf{x}) \cdot \mathbf{t}\right) \mathrm{d} x \\
& =-\frac{1}{2}\left(\beta \mathbf{n} \cdot \boldsymbol{\sigma}^{(2)} \cdot \mathbf{n}+\gamma \mathbf{n} \cdot \boldsymbol{\sigma}^{(2)} \cdot \mathbf{t}\right)
\end{aligned}
$$

for which: $\sigma^{(2)}(\mathbf{x})=\sigma^{(2)}=$ const, $\forall \mathbf{x} \in \omega$ for a dilute concentration of cracks.

Final expression of the free energy $\mathscr{W}$ of the microcracked material with open cracks parallel to $\mathbf{i}_{1}$ direction (for further details refer to $[20,21]$ ) is:

$$
\mathscr{W}=\mathscr{W}_{0}-d\left[H_{n n}(\mathbf{N}: \mathbf{E})^{2}+H_{t t}(\mathbf{T}: \mathbf{E})^{2}\right]
$$


with

$$
\mathscr{W}_{0}=\frac{1}{2} \mathbf{E}: \mathbb{C}_{0}: \mathbf{E}
$$

be the overall free energy of the virgin initially-orthotropic material and $d=\mathscr{N} l^{2}$ be the microcracks density, where $\mathscr{N}$ is the number of cracks per unit surface area, and as usual, $l$ is the half-length of a crack. Parameters $H_{n n}=C(1+D)$ and $H_{t t}=$ $C(1-D)$ are identical to $B_{n n}$ and $B_{t t}$ respectively, of the stress-based approach of Kachanov type. Constants $C$ and $D$ can be expressed in terms of engineering mechanical parameters, or equivalently, in terms of the components of tensor $\mathbb{C}_{0}$ [21]. Moreover, second-order tensors $\mathbf{N}=\mathbb{C}_{0}:\left(\mathbf{i}_{3} \otimes \mathbf{i}_{3}\right)$ and $\mathbf{T}=\mathbb{C}_{0}:\left(\mathbf{i}_{1} \otimes^{s} \mathbf{i}_{3}\right)$ are used.

Finally, from Eq. (91) the effective stiffness tensor $\mathbb{C}$ of the microcracked material, is obtained.

It is worth noting that all the obtained coefficients $K_{k i}^{j l}$ are of the form $f\left(\mathbb{C}_{0}\right)-$ $d\left[g\left(\mathbb{C}_{0}\right)\right]$ with $f, g$ generic functions. It is well highlighted a shortcoming of this kind of formulation in dilute limit assumption, that is severely limit values of the microcracks density $d$. Nevertheless, a great advantage of such a homogenization can be leading to coefficients which do not depend on the REV geometry.

From a computational point of view, the implementation of a hard interface model, also for a quite simple geometry, is not an easy issue due to the discontinuities both in terms of stresses and displacements at the interface. This aspect is not considered in the present work, nevertheless some numerical results are given in [14].

\section{A St. Venant-Kirchhoff Imperfect Interface Model}

In this section, a nonlinear-imperfect interface model is proposed. Within the framework of the detailed micromechanical approach, the model is formulated following the same procedure detailed in Sect. 2, in order to derive both soft and hard imperfect interface laws. In detail, the matched asymptotic expansion method [1, 4, 38-41, 52, $53]$ is extended to the finite strain theory [14, 15, 49]. Moreover, the homogenization method for microcracked media under the NIA [34, 35, 43, 57, 60] is applied to a damaged interphase comprising of a hyperelastic St. Venant-Kirchhoff initially orthotropic material [49].

\subsection{Matched Asymptotic Expansion Method in Finite Strains}

Let an orthonormal Cartesian basis $\left(O, \mathbf{i}_{1}, \mathbf{i}_{2}, \mathbf{i}_{3}\right)$ be introduced, with $x_{1}, x_{2}$ and $x_{3}$ be the corresponding coordinates of a particle belonging to the system $\Omega^{\varepsilon}$. Refer to Sect. 2 for the notation and three-dimensional problem statement (see Fig. 1). 
The equations governing the equilibrium problem of such a composite system are expressed as follows:

$$
\begin{cases}\left(s_{i j}^{\varepsilon}+s_{k j}^{\varepsilon} u_{i, k}^{\varepsilon}\right)_{, j}+f_{i}=0 & \text { in } \Omega_{ \pm}^{\varepsilon} \\ \left(s_{i j}^{\varepsilon}+s_{k j}^{\varepsilon} u_{i, k}^{\varepsilon}\right) n_{j}=p_{i} & \text { on } \Gamma_{1} \\ \left(s_{i j}^{\varepsilon}+s_{k j}^{\varepsilon} u_{i, k}^{\varepsilon}\right)_{, j}=0 & \text { in } \mathscr{B}^{\varepsilon} \\ {\left[\left[s_{i 3}^{\varepsilon}+s_{k 3}^{\varepsilon} u_{i, k}^{\varepsilon}\right]\right]=0} & \text { on } \mathscr{S}_{ \pm}^{\varepsilon} \\ {\left[\left[u_{i}^{\varepsilon}\right]\right]=0} & \text { on } \mathscr{S}_{ \pm}^{\varepsilon} \\ u_{i}^{\varepsilon}=0 & \text { on } \Gamma_{0} \\ s_{i j}^{\varepsilon}=A_{i j h k}^{ \pm} E_{h k}\left(u^{\varepsilon}\right) & \text { in } \Omega_{ \pm}^{\varepsilon} \\ s_{i j}^{\varepsilon}=A_{i j h k}^{\varepsilon} E_{h k}\left(u^{\varepsilon}\right) & \text { in } \mathscr{B}^{\varepsilon}\end{cases}
$$

where $\mathbf{s}^{\varepsilon}$ is the second Piola-Kirchhoff stress tensor, $\mathbf{E}\left(u^{\varepsilon}\right)$ is the Green-Lagrange strain tensor $\left(E_{i j}\left(u^{\varepsilon}\right)=\frac{1}{2}\left(u_{i, j}+u_{j, i}+u_{k, i} u_{k, j}\right)\right.$ with $\left.i, j=1,2,3\right)$ and $\mathbb{A}^{ \pm}, \mathbb{A}^{\varepsilon}$ are the elasticity tensors of the deformable adherents and of the adhesive, respectively. It is worth remarking that for the elastic tensor $\mathbb{A}^{\varepsilon}$ holds the following identity $\mathbb{A}^{\varepsilon} \equiv \mathbb{B}^{\varepsilon}$. Additionally, by the homogenization for microcracked media, detailed in the next section, the interphase elastic tensor is found to be consistent with the soft interphase assumption. Such a finding, allows to express its components through the following relationship:

$$
A_{i j k l}^{\varepsilon}=\varepsilon \hat{A}_{i j k l}
$$

Since the interphase is assumed to behave as a thin layer of thickness $\varepsilon$, it is natural to seek the solution of the equilibrium problem, expressed by Eq. (93), by using asymptotic expansions with respect to the small parameter $\varepsilon$ [42]. In particular, the following asymptotic series with fractional powers are exploited [54]:

$$
\left\{\begin{array}{l}
\mathbf{u}^{\varepsilon}\left(x_{1}, x_{2}, x_{3}\right)=\mathbf{u}^{0}+\varepsilon^{1 / 3} \mathbf{u}^{1}+\varepsilon^{2 / 3} \mathbf{u}^{2}+\varepsilon \mathbf{u}^{3}+\varepsilon^{4 / 3} \mathbf{u}^{4}+\varepsilon^{5 / 3} \mathbf{u}^{5}+\varepsilon^{2} \mathbf{u}^{6}+o\left(\varepsilon^{2}\right) \\
\mathbf{s}^{\varepsilon}\left(x_{1}, x_{2}, x_{3}\right)=\mathbf{s}^{0}+\varepsilon^{1 / 3} \mathbf{s}^{1}+\varepsilon^{2 / 3} \mathbf{s}^{2}+\varepsilon \mathbf{s}^{3}+\varepsilon^{4 / 3} \mathbf{s}^{4}+\varepsilon^{5 / 3} \mathbf{s}^{5}+\varepsilon^{2} \mathbf{s}^{6}+o\left(\varepsilon^{2}\right)
\end{array}\right.
$$

It is worth remarking that such a choice of a fractional expansion is due to energybased evidences [54]. In particular, from a quite simple mono-dimensional example, proposed in [54], it has been put in evidence that the solution in terms of displacement jump is proportional to $\varepsilon^{\frac{2}{3}}$.

In agreement with [12] and equivalently to what performed in the others models (see Sect. 2), also in this case, let the change of variable $\hat{\mathbf{g}}:\left(x_{1}, x_{2}, x_{3}\right) \rightarrow\left(z_{1}, z_{2}, z_{3}\right)$ be introduced in $\mathscr{B}^{\varepsilon}$, with $z_{1}=x_{1}, z_{2}=x_{2}, z_{3}=x_{3} / \varepsilon$. Moreover, let the change of variable $\overline{\mathbf{g}}:\left(x_{1}, x_{2}, x_{3}\right) \rightarrow\left(z_{1}, z_{2}, z_{3}\right)$ be introduced in $\Omega_{ \pm}^{\varepsilon}$, with $z_{1}=x_{1}, z_{2}=x_{2}$, $z_{3}=x_{3} \pm(1-\varepsilon) / 2$. As a result, the interphase $\mathscr{B}^{\varepsilon}$ and the adherents $\Omega_{ \pm}^{\varepsilon}$ are scaled in domains of unitary thickness $\mathscr{B}$ and $\Omega_{ \pm}$, respectively. In what follows, symbols ${ }^{-}$and $\hat{\imath}$ refer to rescaled quantities for $\mathscr{B}$ and $\Omega_{ \pm}$, respectively. More precisely, $\hat{\mathbf{u}}^{\varepsilon}=\mathbf{u}^{\varepsilon} \circ \hat{\mathbf{g}}^{-1}$ and $\hat{\mathbf{s}}^{\varepsilon}=\mathbf{s}^{\varepsilon} \circ \hat{\mathbf{g}}^{-1}$ denote displacement and stress fields for $\mathscr{B}$, and $\overline{\mathbf{u}}^{\varepsilon}=\mathbf{u}^{\varepsilon} \circ \overline{\mathbf{g}}^{-1}$ and $\overline{\mathbf{s}}^{\varepsilon}=\mathbf{s}^{\varepsilon} \circ \overline{\mathbf{g}}^{-1}$ are displacement vector and stress tensor for $\Omega_{ \pm}$, $\mathbf{u}^{\varepsilon}$ and $\mathbf{s}^{\varepsilon}$ being the corresponding fields on the system $\Omega^{\varepsilon}$. The internal and external forces, $\mathbf{f}$ and $\mathbf{p}$, respectively, are assumed to be independent of $\varepsilon$. As a consequence, it 
is set $\overline{\mathbf{f}}\left(z_{1}, z_{2}, z_{3}\right)=\mathbf{f}\left(x_{1}, x_{2}, x_{3}\right)$ and $\overline{\mathbf{p}}\left(z_{1}, z_{2}, z_{3}\right)=\mathbf{p}\left(x_{1}, x_{2}, x_{3}\right)$. Moreover, under the change of variables, the domains $\Gamma_{0}$ and $\Gamma_{1}$ are transformed into the domains denoted by $\bar{\Gamma}_{0}$ and $\bar{\Gamma}_{1}$, respectively. As a result, the governing equations of the equilibrium problem, in the rescaled composite system, are expressed as follows:

$$
\begin{cases}\left(\bar{s}_{i j}+\bar{s}_{k j} \bar{u}_{i, k}\right)_{, j}+\bar{f}_{i}=0 & \text { in } \Omega_{ \pm} \\ \left(\bar{s}_{i j}+\bar{s}_{k j} \bar{u}_{i, k}\right) n_{j}=\bar{p}_{i} & \text { on } \bar{\Gamma}_{1} \\ \left(\hat{s}_{i \alpha}+\hat{s}_{k \alpha} \hat{u}_{i, k}\right)_{, \alpha}+\frac{1}{\varepsilon}\left(\hat{s}_{i 3}+\hat{s}_{k 3} \hat{u}_{i, k}\right)_{, 3}=0 & \text { in } \mathscr{B} \\ \bar{s}_{i 3}+\bar{s}_{k 3} \bar{u}_{i, k}=\hat{s}_{i 3}+\hat{s}_{\alpha 3} \hat{u}_{i, \alpha}+\frac{1}{\varepsilon} \hat{s}_{33} \hat{u}_{i, 3} & \text { on } \mathscr{S}_{ \pm} \\ \bar{u}_{i}=\hat{u}_{i} & \text { on } \mathscr{S}_{ \pm} \\ \bar{u}_{i}=0 & \text { on } \bar{\Gamma}_{0} \\ \bar{s}_{i j}=A_{i j h k}^{ \pm} \bar{E}_{h k}(\bar{u}) & \text { in } \Omega_{ \pm} \\ \hat{s}_{i j}=A_{i j h k}^{\varepsilon} \hat{E}_{h k}(\hat{u}) & \text { in } \mathscr{B}\end{cases}
$$

where $\overline{\mathbf{E}}, \hat{\mathbf{E}}$ denote the rescaled Green-Lagrange strain tensors in the adherents and in the adhesive.

In view of Eq. (95) the relevant fields, in the rescaled adhesive and adherents, can be expressed as asymptotic expansions in the following way:

$$
\left\{\begin{array}{l}
\hat{\mathbf{s}}^{\varepsilon}\left(z_{1}, z_{2}, z_{3}\right)=\hat{\mathbf{s}}^{0}+\varepsilon^{1 / 3} \hat{\mathbf{s}}^{1}+\varepsilon^{2 / 3} \hat{\mathbf{s}}^{2}+\varepsilon \hat{\mathbf{s}}^{3}+\varepsilon^{4 / 3} \hat{\mathbf{s}}^{4}+\varepsilon^{5 / 3} \hat{\mathbf{s}}^{5}+\varepsilon^{2} \hat{\mathbf{s}}^{6}+o\left(\varepsilon^{2}\right) \\
\overline{\mathbf{s}}^{\varepsilon}\left(z_{1}, z_{2}, z_{3}\right)=\overline{\mathbf{s}}^{0}+\varepsilon^{1 / 3} \overline{\mathbf{s}}^{1}+\varepsilon^{2 / 3} \overline{\mathbf{s}}^{2}+\varepsilon \overline{\mathbf{s}}^{3}+\varepsilon^{4 / 3} \overline{\mathbf{s}}^{4}+\varepsilon^{5 / 3} \overline{\mathbf{s}}^{5}+\varepsilon^{2} \overline{\mathbf{s}}^{6}+o\left(\varepsilon^{2}\right) \\
\hat{\mathbf{u}}^{\varepsilon}\left(z_{1}, z_{2}, z_{3}\right)=\hat{\mathbf{u}}^{0}+\varepsilon^{1 / 3} \hat{\mathbf{u}}^{1}+\varepsilon^{2 / 3} \hat{\mathbf{u}}^{2}+\varepsilon \hat{\mathbf{u}}^{3}+\varepsilon^{4 / 3} \hat{\mathbf{u}}^{4}+\varepsilon^{5 / 3} \hat{\mathbf{u}}^{5}+\varepsilon^{2} \hat{\mathbf{u}}^{6}+o\left(\varepsilon^{2}\right) \\
\overline{\mathbf{u}}^{\varepsilon}\left(z_{1}, z_{2}, z_{3}\right)=\overline{\mathbf{u}}^{0}+\varepsilon^{1 / 3} \overline{\mathbf{u}}^{1}+\varepsilon^{2 / 3} \overline{\mathbf{u}}^{2}+\varepsilon \overline{\mathbf{u}}^{3}+\varepsilon^{4 / 3} \overline{\mathbf{u}}^{4}+\varepsilon^{5 / 3} \overline{\mathbf{u}}^{5}+\varepsilon^{2} \overline{\mathbf{u}}^{6}+o\left(\varepsilon^{2}\right)
\end{array}\right.
$$

In the following, the conditions holding in the rescaled interphase $\mathscr{B}$ are detailed. These latter are obtained by substituting the first of Eq. (97) into the equilibrium equation holding in the interphase (i.e., third equation of the system (96)) and by identifying, in a standard way, similar terms with respect to the power of the parameter $\varepsilon$ :

- Power of $\varepsilon:-2$

$$
\left(\hat{u}_{i, 3}^{0} \hat{s}_{33}^{0}\right)_{, 3}=0
$$

- Power of $\varepsilon:-5 / 3$

$$
\left(\hat{u}_{i, 3}^{0} \hat{s}_{33}^{1}+\hat{u}_{i, 3}^{1} \hat{s}_{33}^{0}\right)_{, 3}=0
$$

- Power of $\varepsilon:-4 / 3$

$$
\left(\hat{u}_{i, 3}^{0} \hat{s}_{33}^{2}+\hat{u}_{i, 3}^{1} \hat{s}_{33}^{1}+\hat{u}_{i, 3}^{2} \hat{s}_{33}^{0}\right)_{, 3}=0,
$$

- Power of $\varepsilon:-1$

$$
\begin{aligned}
& \left(\hat{u}_{i, 3}^{0} \hat{s}_{3 \alpha}^{0}\right)_{, \alpha}+\left(\hat{s}_{i 3}^{0}+\hat{u}_{i, \alpha}^{0} \hat{s}_{3 \alpha}^{0}\right)_{, 3} \\
& \quad+\left(\hat{u}_{i, 3}^{0} \hat{s}_{33}^{3}+\hat{u}_{i, 3}^{1} \hat{s}_{33}^{2}+\hat{u}_{i, 3}^{2} \hat{s}_{33}^{1}+\hat{u}_{i, 3}^{3} \hat{s}_{33}^{0}\right)_{, 3}=0,
\end{aligned}
$$


- Power of $\varepsilon:-2 / 3$

$$
\begin{aligned}
& \left(\hat{u}_{i, 3}^{0} \hat{s}_{3 \alpha}^{1}+\hat{u}_{i, 3}^{1} \hat{s}_{3 \alpha}^{0}\right)_{, \alpha}+\left(\hat{s}_{i 3}^{1}+\hat{u}_{i, \alpha}^{0} \hat{s}_{3 \alpha}^{1}+\hat{u}_{i, \alpha}^{1} \hat{s}_{3 \alpha}^{0}\right)_{, 3} \\
& \quad+\left(\hat{u}_{i, 3}^{0} \hat{s}_{33}^{4}+\hat{u}_{i, 3}^{1} \hat{s}_{33}^{3}+\hat{u}_{i, 3}^{2} \hat{s}_{33}^{2}+\hat{u}_{i, 3}^{3} \hat{s}_{33}^{1}+\hat{u}_{i, 3}^{4} \hat{s}_{33}^{0}\right)_{, 3}=0
\end{aligned}
$$

- Power of $\varepsilon:-1 / 3$

$$
\begin{aligned}
& \left(\hat{u}_{i, 3}^{0} \hat{s}_{3 \alpha}^{2}+\hat{u}_{i, 3}^{1} \hat{s}_{3 \alpha}^{1}+\hat{u}_{i, 3}^{2} \hat{s}_{3 \alpha}^{0}\right)_{, \alpha} \\
& \quad+\left(\hat{s}_{i 3}^{2}+\hat{u}_{i, \alpha}^{0} \hat{s}_{3 \alpha}^{2}+\hat{u}_{i, \alpha}^{1} \hat{s}_{3 \alpha}^{1}+\hat{u}_{i, \alpha}^{2} \hat{s}_{3 \alpha}^{0}\right)_{, 3} \\
& \quad+\left(\hat{u}_{i, 3}^{0} \hat{s}_{33}^{5}+\hat{u}_{i, 3}^{1} \hat{s}_{33}^{4}+\hat{u}_{i, 3}^{2} \hat{s}_{33}^{3}+\hat{u}_{i, 3}^{3} \hat{s}_{33}^{2}+\hat{u}_{i, 3}^{4} \hat{s}_{33}^{1}+\hat{u}_{i, 3}^{5} \hat{s}_{33}^{0}\right)_{, 3}=0,
\end{aligned}
$$

- Power of $\varepsilon: 0$

$$
\begin{aligned}
& \left(\hat{u}_{i, 3}^{0} \hat{s}_{3 \alpha}^{3}+\hat{u}_{i, 3}^{1} \hat{s}_{3 \alpha}^{2}+\hat{u}_{i, 3}^{2} \hat{s}_{3 \alpha}^{1}+\hat{u}_{i, 3}^{3} \hat{s}_{3 \alpha}^{0}\right)_{, \alpha}+\left(\hat{s}_{i \alpha}^{0}+\hat{s}_{\alpha \beta}^{0} \hat{u}_{i, \beta}^{0}\right)_{\alpha} \\
& \quad+\left(\hat{s}_{i 3}^{3}+\hat{u}_{i, \alpha}^{0} \hat{s}_{3 \alpha}^{3}+\hat{u}_{i, \alpha}^{1} \hat{s}_{3 \alpha}^{2}+\hat{u}_{i, \alpha}^{2} \hat{s}_{3 \alpha}^{1}+\hat{u}_{i, \alpha}^{3} \hat{s}_{3 \alpha}^{0}\right)_{, 3} \\
& \quad+\left(\hat{u}_{i, 3}^{0} \hat{s}_{33}^{6}+\hat{u}_{i, 3}^{1} \hat{s}_{33}^{5}+\hat{u}_{i, 3}^{2} \hat{s}_{33}^{4}+\hat{u}_{i, 3}^{3} \hat{s}_{33}^{3}+\hat{u}_{i, 3}^{4} \hat{s}_{33}^{2}+\hat{u}_{i, 3}^{5} \hat{s}_{33}^{1}+\hat{u}_{i, 3}^{6} \hat{s}_{33}^{0}\right)_{, 3}=0
\end{aligned}
$$

By substituting the first two equations of (97) into the continuity condition of the traction vector holding through the rescaled interfaces $\mathscr{S}_{ \pm}$(i.e., fourth equation of system (96)), and by applying the usual identification procedure, the following relationships are obtained:

- Power of $\varepsilon:-1$

$$
0=\left(\hat{u}_{i, 3}^{0} \hat{s}_{33}^{0}\right)
$$

- Power of $\varepsilon:-2 / 3$

$$
0=\left(\hat{u}_{i, 3}^{0} \hat{s}_{33}^{1}+\hat{u}_{i, 3}^{1} \hat{s}_{33}^{0}\right)
$$

- Power of $\varepsilon:-1 / 3$

$$
0=\left(\hat{u}_{i, 3}^{0} \hat{s}_{33}^{2}+\hat{u}_{i, 3}^{1} \hat{s}_{33}^{1}+\hat{u}_{i, 3}^{2} \hat{s}_{33}^{0}\right)
$$

- Power of $\varepsilon: 0$

$$
\left(\bar{s}_{i 3}^{0}+\bar{u}_{i k}^{0} \bar{s}_{k 3}^{0}\right)=\left(\hat{s}_{i 3}^{0}+\hat{u}_{i, \alpha}^{0} \hat{s}_{\alpha 3}^{0}+\hat{u}_{i, 3}^{0} \hat{s}_{33}^{3}+\hat{u}_{i, 3}^{1} \hat{s}_{33}^{2}+\hat{u}_{i, 3}^{2} \hat{s}_{33}^{1}+\hat{u}_{i, 3}^{3} \hat{s}_{33}^{0}\right)
$$


- Power of $\varepsilon: 1 / 3$

$$
\begin{aligned}
& \left(\bar{s}_{i 3}^{1}+\bar{u}_{i, k}^{0} \bar{s}_{k 3}^{1}+\bar{u}_{i, k}^{1} \bar{s}_{k 3}^{0}\right) \\
& =\left(\hat{s}_{i 3}^{1}+\hat{u}_{i, \alpha}^{0} \hat{s}_{\alpha 3}^{1}+\hat{u}_{i, \alpha}^{1} \hat{s}_{\alpha 3}^{0}\right) \\
& \quad+\left(\hat{u}_{i, 3}^{0} \hat{s}_{33}^{4}+\hat{u}_{i, 3}^{1} \hat{s}_{33}^{3}\right. \\
& \left.\quad+\hat{u}_{i, 3}^{2} \hat{s}_{33}^{2}+\hat{u}_{i, 3}^{3} \hat{s}_{33}^{1}+\hat{u}_{i, 3}^{4} \hat{s}_{33}^{0}\right)
\end{aligned}
$$

- Power of $\varepsilon: 2 / 3$

$$
\begin{aligned}
& \left(\bar{s}_{i 3}^{2}+\bar{u}_{i, k}^{0} \bar{s}_{k 3}^{2}+\bar{u}_{i, k}^{1} \bar{s}_{k 3}^{1}+\bar{u}_{i, k}^{2} \bar{s}_{k 3}^{0}\right) \\
& \quad=\left(\hat{s}_{33}^{2}+\hat{u}_{i, \alpha}^{0} \hat{s}_{\alpha 3}^{2}+\hat{u}_{i, \alpha}^{1} \hat{s}_{\alpha 3}^{1}+\hat{u}_{i, \alpha}^{0} \hat{s}_{\alpha 3}^{2}\right) \\
& \quad+\left(\hat{u}_{i, 3}^{0} \hat{s}_{33}^{5}+\hat{u}_{i, 3}^{1} \hat{s}_{33}^{4}+\hat{u}_{i, 3}^{2} \hat{s}_{33}^{3}+\hat{u}_{i, 3}^{3} \hat{s}_{33}^{2}+\hat{u}_{i, 3}^{4} \hat{s}_{33}^{1}+\hat{u}_{i, 3}^{5} \hat{s}_{33}^{0}\right)
\end{aligned}
$$

- Power of $\varepsilon: 1$

$$
\begin{aligned}
\left(\bar{s}_{i 3}^{3}+\right. & \left.\bar{u}_{i, k}^{0} \bar{s}_{k 3}^{3}+\bar{u}_{i, k}^{1} \bar{s}_{k 3}^{2}+\bar{u}_{i, k}^{2} \bar{s}_{k 3}^{1}+\bar{u}_{i, k}^{3} \bar{s}_{k 3}^{0}\right) \\
= & \left(\hat{s}_{i 3}^{3}+\hat{u}_{i, \alpha}^{0} \hat{s}_{\alpha 3}^{3}+\hat{u}_{i, \alpha}^{1} \hat{s}_{\alpha 3}^{2}+\hat{u}_{i, \alpha}^{2} \hat{s}_{\alpha 3}^{1}+\hat{u}_{i, \alpha}^{3} \hat{s}_{\alpha 3}^{0}\right) \\
& \quad+\left(\hat{u}_{i, 3}^{0} \hat{s}_{33}^{6}+\hat{u}_{i, 3}^{1} \hat{s}_{33}^{5}+\hat{u}_{i, 3}^{2} \hat{s}_{33}^{4}+\hat{u}_{i, 3}^{3} \hat{s}_{33}^{3}+\hat{u}_{i, 3}^{4} \hat{s}_{33}^{2}+\hat{u}_{i, 3}^{5} \hat{s}_{33}^{1}+\hat{u}_{i, 3}^{6} \hat{s}_{33}^{0}\right)
\end{aligned}
$$

- ...

It is worth noting that the above equations hold both in $\mathscr{S}_{+}$and in $\mathscr{S}_{-}$, for the sake of briefness they have been detailed only in one case. Moreover, by remarking that the left-hand sides in Eqs.(108)-(111) can be identified as the expansions of the $\mathbf{i}_{3}$ components of the first Piola-Kirchhoff stress tensor $\bar{P}_{i 3}=\left(\bar{s}_{i 3}+\bar{u}_{i k} \bar{s}_{k 3}\right)$ in the adherents, a significant simplification of these equations it is possible.

According to the soft-material-interphase assumption, by substituting Eq. (94) in the constitutive law holding in the interphase $\mathscr{B}$ of the rescaled domain (i.e., last equation of the problem (96)), written for $j=3$, the following conditions are deduced:

- Power of $\varepsilon:-1$

$$
0=\left(\hat{u}_{k, 3}^{0} \hat{u}_{k, 3}^{0}\right)
$$

- Power of $\varepsilon:-2 / 3$

$$
0=\left(\hat{u}_{k, 3}^{0} \hat{u}_{k, 3}^{1}+\hat{u}_{k, 3}^{1} \hat{u}_{k, 3}^{0}\right)
$$

- Power of $\varepsilon:-1 / 3$

$$
0=\left(\hat{u}_{k, 3}^{0} \hat{u}_{k, 3}^{2}+\hat{u}_{k, 3}^{1} \hat{u}_{k, 3}^{1}+\hat{u}_{k, 3}^{2} \hat{u}_{k, 3}^{0}\right)
$$


- Power of $\varepsilon: 0$

$$
\begin{aligned}
& \hat{s}_{\alpha 3}^{0}=\hat{A}_{33 \alpha 3}\left[\hat{u}_{3,3}^{0}+\left(\hat{u}_{s, 3}^{0} \hat{u}_{s, 3}^{3}+\hat{u}_{s, 3}^{1} \hat{u}_{s, 3}^{2}\right)\right]+\frac{1}{2} \hat{A}_{\beta 3 \alpha 3}\left(\hat{u}_{\beta, 3}^{0}+\hat{u}_{s, \beta}^{0} \hat{u}_{s, 3}^{0}\right) \\
& \hat{s}_{33}^{0}=\hat{A}_{3333}\left[\hat{u}_{3,3}^{0}+\left(\hat{u}_{s, 3}^{0} \hat{u}_{s, 3}^{3}+\hat{u}_{s, 3}^{1} \hat{u}_{s, 3}^{2}\right)\right]+\frac{1}{2} \hat{A}_{33 \beta 3}\left(\hat{u}_{\beta, 3}^{0}+\hat{u}_{s, \beta}^{0} \hat{u}_{s, 3}^{0}\right)
\end{aligned}
$$

- Power of $\varepsilon: 1 / 3$

$$
\begin{aligned}
\hat{s}_{\alpha 3}^{1}= & \hat{A}_{33 \alpha 3}\left[\hat{u}_{3,3}^{1}+\left(\hat{u}_{s, 3}^{0} \hat{u}_{s, 3}^{4}+\hat{u}_{s, 3}^{1} \hat{u}_{s, 3}^{3}+\frac{1}{2} \hat{u}_{s, 3}^{2} \hat{u}_{s, 3}^{2}\right)\right] \\
& +\frac{1}{2} \hat{A}_{\beta 3 \alpha 3}\left(\hat{u}_{\beta, 3}^{1}+\hat{u}_{s, \beta}^{0} \hat{u}_{s, 3}^{1}+\hat{u}_{s, \beta}^{1} \hat{u}_{s, 3}^{0}\right) \\
\hat{s}_{33}^{1}= & \hat{A}_{3333}\left[\hat{u}_{3,3}^{1}+\left(\hat{u}_{s, 3}^{0} \hat{u}_{s, 3}^{4}+\hat{u}_{s, 3}^{1} \hat{u}_{s, 3}^{3}+\frac{1}{2} \hat{u}_{s, 3}^{2} \hat{u}_{s, 3}^{2}\right)\right] \\
& +\frac{1}{2} \hat{A}_{33 \beta 3}\left(\hat{u}_{\beta, 3}^{1}+\hat{u}_{s, \beta}^{0} \hat{u}_{s, 3}^{1}+\hat{u}_{s, \beta}^{1} \hat{u}_{s, 3}^{0}\right)
\end{aligned}
$$

From Eqs. (112)-(115) it follows that:

$$
\begin{aligned}
& \hat{\mathbf{u}}_{, 3}^{0}=0 \text { in } \mathscr{B} \Rightarrow\left[\hat{\mathbf{u}}^{0}\right]=0 \\
& \hat{\mathbf{u}}_{, 3}^{1}=0 \text { in } \mathscr{B} \Rightarrow\left[\hat{\mathbf{u}}^{1}\right]=0 \\
& \hat{s}_{\alpha 3}^{0}=0=\hat{s}_{33}^{0} \text { in } \mathscr{B}
\end{aligned}
$$

where it is set $[f]\left(z_{1}, z_{2}\right)=f\left(z_{1}, z_{2}, 1 / 2\right)-f\left(z_{1}, z_{2},-1 / 2\right)$ for $f: \mathscr{B} \mapsto \mathbb{R}^{3}$. By combining Eqs.(117)-(119) into Eqs. (98)-(101), the following relationship is obtained:

$$
\left(\hat{u}_{i, 3}^{2} \hat{s}_{33}^{1}\right)_{3}=0 \text { in } \mathscr{B}
$$

which integrated with respect to $z_{3}$ gives

$$
\hat{u}_{i, 3}^{2} \hat{s}_{33}^{1}=\text { const. }=\left.\bar{P}_{i 3}^{0}\right|_{\mathscr{S}_{ \pm}} \text {in } \mathscr{B}
$$

where $\left.\bar{P}_{i 3}^{0}\right|_{\mathscr{S}_{ \pm}}$is the common value taken at the interfaces $\mathscr{S}_{ \pm}$(cfr. Eq. (108)). Moreover, by substituting Eq.(116) and Eqs.(117)-(119) into Eq. (121) the following relationship is obtained:

$$
\frac{1}{2} \hat{A}_{3333}\left(\left|\hat{u}_{i, 3}^{2}\right|^{2} \hat{u}_{i, 3}^{2}\right)=\bar{P}_{i 3}^{0}=\text { in } \mathscr{B}
$$


By solving with respect to $\hat{\mathbf{u}}_{, 3}^{2}$ and by integrating with respect to $z_{3}$ one has:

$$
\left[\hat{\mathbf{u}}^{2}\right]=\frac{1}{\left(\frac{1}{2} \hat{A}_{3333}\right)^{1 / 3}} \frac{1}{\left|\overline{\mathbf{P}}^{0} \mathbf{i}_{3}\right|^{2 / 3}} \overline{\mathbf{P}}^{0} \mathbf{i}_{3}
$$

Thereby, substituting Eqs.(112)-(115) into Eqs.(108)-(110) it is obtained that:

$$
\begin{aligned}
{\left[\overline{\mathbf{P}}^{0} \mathbf{i}_{3}\right] } & =0 \\
{\left[\overline{\mathbf{P}}^{1} \mathbf{i}_{3}\right] } & =0 \\
{\left[\overline{\mathbf{P}}^{2} \mathbf{i}_{3}\right] } & =0
\end{aligned}
$$

with

$$
\begin{aligned}
\bar{P}_{i 3}^{0} & =\left(\bar{s}_{i 3}^{0}+\bar{u}_{i, k}^{0} \bar{s}_{k 3}^{0}\right) \\
\bar{P}_{i 3}^{1} & =\left(\bar{s}_{i 3}^{1}+\bar{u}_{i, k}^{0} \bar{s}_{k 3}^{1}+\bar{u}_{i, k}^{1} \bar{s}_{k 3}^{0}\right) \\
\bar{P}_{i 3}^{2} & =\left(\bar{s}_{i 3}^{2}+\bar{u}_{i, k}^{0} \bar{s}_{k 3}^{2}+\bar{u}_{i, k}^{1} \bar{s}_{k 3}^{1}+\bar{u}_{i, k}^{2} \bar{s}_{k 3}^{0}\right)
\end{aligned}
$$

The final step of the asymptotic expansion method consists in applying the matching conditions in order to find the proper interface law for the limit equilibrium problem, in which the interphase is replaced by the limit interface $\mathscr{S}$ and the adherents by the domains $\Omega_{ \pm}^{0}=\left\{\left(x_{1}, x_{2}, x_{3}\right) \in \Omega: \pm x_{3}>0\right\}$. By taking into account the asymptotic expansion of the displacement field (95) and assuming that $\mathbf{u}^{\varepsilon}$ in the adherent can be expanded in a Taylor series representation along the $x_{3}$-direction, it results:

$$
\begin{aligned}
\mathbf{u}^{\varepsilon}\left(\overline{\mathbf{x}}, \pm \frac{\varepsilon}{2}\right)= & \mathbf{u}^{\varepsilon}\left(\overline{\mathbf{x}}, 0^{ \pm}\right) \pm \frac{\varepsilon}{2} \mathbf{u}_{, 3}^{\varepsilon}\left(\overline{\mathbf{x}}, 0^{ \pm}\right)+\cdots \\
= & \mathbf{u}^{0}\left(\overline{\mathbf{x}}, 0^{ \pm}\right)+\varepsilon^{1 / 3} \mathbf{u}^{1}\left(\overline{\mathbf{x}}, 0^{ \pm}\right)+\varepsilon^{2 / 3} \mathbf{u}^{2}\left(\overline{\mathbf{x}}, 0^{ \pm}\right) \\
& +\varepsilon\left(\mathbf{u}^{3}\left(\overline{\mathbf{x}}, 0^{ \pm}\right) \pm \frac{1}{2} \mathbf{u}_{, 3}^{0}\left(\overline{\mathbf{x}}, 0^{ \pm}\right)\right)+\cdots
\end{aligned}
$$

In view of the continuity of the displacements at the interfaces $\mathscr{S}_{ \pm}^{\varepsilon}$ and $\mathscr{S}_{ \pm}$one has

$$
\begin{aligned}
& \mathbf{u}^{0}\left(\overline{\mathbf{x}}, 0^{ \pm}\right)+\varepsilon^{1 / 3} \mathbf{u}^{1}\left(\overline{\mathbf{x}}, 0^{ \pm}\right)+\varepsilon^{2 / 3} \mathbf{u}^{2}\left(\overline{\mathbf{x}}, 0^{ \pm}\right)+\cdots \\
& =\hat{\mathbf{u}}^{0}\left(\overline{\mathbf{z}}, \pm \frac{1}{2}\right)+\varepsilon^{1 / 3} \hat{\mathbf{u}}^{1}\left(\overline{\mathbf{z}}, \pm \frac{1}{2}\right)+\cdots \\
& =\overline{\mathbf{u}}^{0}\left(\overline{\mathbf{z}}, \pm \frac{1}{2}\right)+\varepsilon^{1 / 3} \overline{\mathbf{u}}^{1}\left(\overline{\mathbf{z}}, \pm \frac{1}{2}\right)+\cdots
\end{aligned}
$$

and, identifying the terms in the same powers of $\varepsilon$, it is deduced that: 


$$
\begin{aligned}
& \mathbf{u}^{0}\left(\overline{\mathbf{x}}, 0^{ \pm}\right)=\hat{\mathbf{u}}^{0}\left(\overline{\mathbf{z}}, \pm \frac{1}{2}\right)=\overline{\mathbf{u}}^{0}\left(\overline{\mathbf{z}}, \pm \frac{1}{2}\right) \\
& \mathbf{u}^{1}\left(\overline{\mathbf{x}}, 0^{ \pm}\right)=\hat{\mathbf{u}}^{1}\left(\overline{\mathbf{z}}, \pm \frac{1}{2}\right)=\overline{\mathbf{u}}^{1}\left(\overline{\mathbf{z}}, \pm \frac{1}{2}\right) \\
& \mathbf{u}^{2}\left(\overline{\mathbf{x}}, 0^{ \pm}\right)=\hat{\mathbf{u}}^{2}\left(\overline{\mathbf{z}}, \pm \frac{1}{2}\right)=\overline{\mathbf{u}}^{2}\left(\overline{\mathbf{z}}, \pm \frac{1}{2}\right)
\end{aligned}
$$

Analogous results can be obtained for the tractions vector, herein expressed in terms of the first Piola-Kirchhoff tensor:

$$
\begin{aligned}
& \mathbf{P}^{0}\left(\overline{\mathbf{x}}, 0^{ \pm}\right) \mathbf{i}_{3}=\hat{\mathbf{P}}^{0}\left(\overline{\mathbf{z}}, \pm \frac{1}{2}\right) \mathbf{i}_{3}=\overline{\mathbf{P}}^{0}\left(\overline{\mathbf{z}}, \pm \frac{1}{2}\right) \mathbf{i}_{3} \\
& \mathbf{P}^{1}\left(\overline{\mathbf{x}}, 0^{ \pm}\right) \mathbf{i}_{3}=\hat{\mathbf{P}}^{1}\left(\overline{\mathbf{z}}, \pm \frac{1}{2}\right) \mathbf{i}_{3}=\overline{\mathbf{P}}^{1}\left(\overline{\mathbf{z}}, \pm \frac{1}{2}\right) \mathbf{i}_{3} \\
& \mathbf{P}^{2}\left(\overline{\mathbf{x}}, 0^{ \pm}\right) \mathbf{i}_{3}=\hat{\mathbf{P}}^{2}\left(\overline{\mathbf{z}}, \pm \frac{1}{2}\right) \mathbf{i}_{3}=\overline{\mathbf{P}}^{2}\left(\overline{\mathbf{z}}, \pm \frac{1}{2}\right) \mathbf{i}_{3}
\end{aligned}
$$

Let the following notation be adopted: $[[\mathbf{f}]]:=\mathbf{f}\left(\mathbf{x}, 0^{+}\right)-\mathbf{f}\left(\mathbf{x}, 0^{-}\right)$with $\mathbf{f}$ : $\Omega_{+}^{0} \cup \Omega_{-}^{0} \mapsto \mathbb{R}^{3}$; accordingly, the proper contact conditions for the limit equilibrium problem, i.e. expressed in terms of the relevant fields defined on $\Omega_{+}^{0} \cup \Omega_{-}^{0}$, can be obtained by using this relation into the interphase laws Eqs. (117), (118), (123), (124)-(126):

$$
\begin{aligned}
{\left[\overline{\mathbf{u}}^{l}\right] } & =\left[\left[\mathbf{u}^{l}\right]\right] \quad l=0,1,2 \\
{\left[\overline{\mathbf{P}}^{l} \mathbf{i}_{3}\right] } & =\left[\left[\mathbf{P}^{l} \mathbf{i}_{3}\right]\right] \quad l=0,1,2
\end{aligned}
$$

By applying the matching relations (134) and taking into account Eqs. (132) and (133), the interface laws for the soft interphase can be rewritten in the limit configuration $\Omega_{+}^{0} \cup \Omega_{-}^{0} \cup \mathscr{S}$ in a form involving only the fields in the adherents:

$$
\begin{gathered}
{\left[\left[\mathbf{u}^{0}\right]\right]=0 \quad\left[\left[\mathbf{P}^{0} \mathbf{i}_{3}\right]\right]=0} \\
{\left[\left[\mathbf{u}^{1}\right]\right]=0 \quad\left[\left[\mathbf{P}^{1} \mathbf{i}_{3}\right]\right]=0} \\
{\left[\left[\mathbf{u}^{2}\right]\right]=\frac{1}{\left(\frac{1}{2} \hat{A}_{3333}\right)^{1 / 3}} \frac{1}{\left|\mathbf{P}^{0} \mathbf{i}_{3}\right|^{2 / 3}} \mathbf{P}^{0} \mathbf{i}_{3} \quad\left[\left[\mathbf{P}^{2} \mathbf{i}_{3}\right]\right]=0}
\end{gathered}
$$

which are the final expressions of the interface conditions for the proposed St. VenantKirchhoff anisotropic model. It is worth remarking that the imperfect interface condition, prescribing a jump of the displacement, appears at the second order. By taking into account the expansions (95) and the relations (127)-(129), one finds: 


$$
\begin{aligned}
\mathbf{P}^{\varepsilon} \mathbf{i}_{3} & =\mathbf{P}^{0} \mathbf{i}_{3}+O\left(\varepsilon^{1 / 3}\right) \\
{\left[\left[\mathbf{P}^{\varepsilon} \mathbf{i}_{3}\right]\right] } & =\varepsilon^{2 / 3}\left[\left[\mathbf{P}^{2} \mathbf{i}_{3}\right]\right]+O(\varepsilon) \\
{\left[\left[\mathbf{u}^{\varepsilon}\right]\right] } & =\varepsilon^{2 / 3}\left[\left[\mathbf{u}^{2}\right]\right]+O(\varepsilon)
\end{aligned}
$$

which, substituted into (135)-(137), give

$$
\begin{aligned}
& {\left[\left[\mathbf{P}^{\varepsilon} \mathbf{i}_{3}\right]\right]=0+o(\varepsilon)} \\
& \mathbf{P}^{\varepsilon} \mathbf{i}_{3}=\frac{A_{3333}^{\varepsilon}}{2 \varepsilon^{3}}\left|\left[\left[\mathbf{u}^{\varepsilon}\right]\right]\right|^{2}\left[\left[\mathbf{u}^{\varepsilon}\right]\right]+o\left(\varepsilon^{1 / 3}\right)
\end{aligned}
$$

Note that, to the aim to fully express the interface law Eq. (142) within the interphase domain, Eq. (94) is taken into account: $\hat{A}_{3333}=\frac{A_{3333}^{\varepsilon}}{\varepsilon}$.

Finally, the imperfect interface law can be rewritten in terms of the Piola stress vector $\mathbf{P} \mathbf{i}_{3}$ and the displacement jump [[u]] in the limit configuration (Fig. 1c):

$$
\mathbf{P i}_{3}=\frac{\hat{A}_{3333}}{2 \varepsilon^{2}}|[[\mathbf{u}]]|^{2}[[\mathbf{u}]]
$$

Remark that Eq. (143) is the relevant expression, from a computational point of view, of the interface law for the proposed St. Venant-Kirchhoff model. Thereby, it represents the transmission condition for the stress vector $\mathbf{P} \mathbf{i}_{3}$ across the interface $\mathscr{S}$. As a definition, $\hat{A}_{3333}$ is the interface stiffness for this soft nonlinear interface. Moreover, Eq. (143) highlights that this transmission condition is an effectively nonlinear imperfect interface law. It is worth remarking that such an imperfect interface condition, in order to be numerically implemented needs to fix a value for the thickness $\varepsilon$. This fact can represent a shortcoming for the proposed model. Nevertheless, such a parameter, in many cases can be measurable, for instance in the case of the glue layers in bonding problems.

\subsection{Homogenization of the Microcracked Interphase}

The interface law (see Eq. 142) is a function of the elastic constant $A_{3333}^{\varepsilon}$ of the interphase material. This latter is assumed to be orthotropic with principal axes $\left(\mathbf{i}_{1}, \mathbf{i}_{3}\right)$ and weakened by one family of parallel rectilinear microcracks with length $2 l$ and orientation $\phi=\left(\mathbf{i}_{1}, \mathbf{t}\right)=0^{\circ}$. In order to recover the elastic constant $A_{3333}^{\varepsilon}$ and consecutively the interface stiffness $\hat{A}_{3333}$, the homogenization technique for microcracked orthotropic media in the two-dimensional context, refer to [17] for further details. Therefore, in what follows only the relevant relations are outlined.

Recall the expression of the effective compliance tensor $\mathbb{S}$ of the microcracked interphase, obtained through a stress-based homogenization in NIA:

$$
(\mathbb{S})_{i j k l}=\left(\mathbb{S}_{0}\right)_{i j k l}+(\Delta \mathbb{S})_{i j k l}
$$


where $\Delta \mathbb{S}$ is the contribution compliance tensor associated to the perturbative term in the complementary elastic potential $\Delta f$, and accounting for the crack features. $\mathbb{S}_{0}$ is the compliance of the undamaged initially orthotropic interphase material. As a result, the elasticity tensor $\mathbb{A}^{\varepsilon}$ can be easily derived as: $\mathbb{A}^{\varepsilon}=\mathbb{S}^{-1}$. Note that the tensor $\mathbb{A}^{\varepsilon}$ depends on the interphase thickness $\varepsilon$ through the microcrack density $\rho$ :

$$
\rho=\frac{l^{2}}{|R E A|}=\frac{l^{2}}{\varepsilon L}
$$

with $L$ a characteristic dimension of the interphase, and $\varepsilon$ is the interphase thickness, as usual. As a result, the elastic constant $A_{3333}^{\varepsilon}$ reads as:

$$
A_{3333}^{\varepsilon}=\frac{E_{1} E_{3}}{E_{1}+2 B_{n n} \rho E_{1} E_{3}-E_{3} v_{13}^{2}}
$$

with

$$
B_{n n}=\frac{\pi}{2 \sqrt{E_{3}}} \sqrt{\frac{2}{\sqrt{E_{1} E_{3}}}+\frac{1}{G_{13}}-\frac{2 v_{13}}{E_{1}}}
$$

where $E_{1}, E_{3}, G_{13}$ and $v_{13}$ are the elastic constants of the undamaged interphase. It is worth pointing out that these latter can be obtained in terms of the elastic properties of the two adherents, as the result of a homogenization step performed on the undamaged $\varepsilon$-thick representative elementary volume $[17,50,51]$.

Finally, the interface stiffness $\hat{A}_{3333}$, derived from Eq. (146), is expressed by the following relationship:

$$
\hat{A}_{3333}=\frac{L}{2 B_{n n} l^{2}}
$$

\section{Numerical Applications}

In this section a numerical benchmark is proposed in order to validate the imperfectnonlinear-interface model of the St. Venant-Kirchhoff type detailed in Sect. 3 and to compare it with the spring-like model described in Sect.2. A quite simple threedimensional geometry is treated, in particular an unit brick $(210 \mathrm{~mm} \times 100 \mathrm{~mm} \times$ $50 \mathrm{~mm})$ joined with a mortar joint $(210 \mathrm{~mm} \times 100 \mathrm{~mm} \times 10 \mathrm{~mm})$. The composite system is assumed to be fixed on a flat rigid plane. The geometry and the boundary conditions are outlined in Fig. 3. This simple academic model has been chosen to focus, in a more accurate way, on the behavior of the brick/mortar interface.

With respect to the hypothesis on the constitutive behavior of the principal constituents, i.e. brick and mortar, a linear and a nonlinear isotropic cases have been distinguished. In the first case, the materials are assumed to be linearly elastic with parameters: Young modulus $E_{b}=13 \times 10^{3} \mathrm{MPa}$ and Poisson ratio $v_{b}=0.2$ for the brick, and Young modulus $E_{m}=4 \times 10^{3} \mathrm{MPa}$ and Poisson ratio $v_{m}=0.2$ for the 

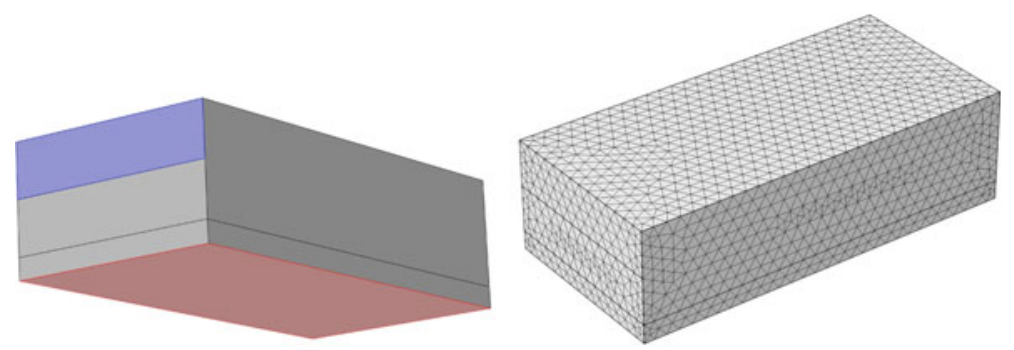

Fig. 3 Geometry, boundary conditions and mesh detail—sketch of the three-dimensional model (on the left). The surface loaded with the incremental displacement is represented in blue and the red surface is fixed. On the right side, a detail of the free tetrahedral mesh is represented (color online)

mortar, respectively. Instead, in the nonlinear case, both brick and mortar behave as hyperelastic materials of the St. Venant-Kirchhoff type with Lamé constants: $\lambda_{b}=$ $3.6 \times 10^{3} \mathrm{MPa}$ and $\mu_{b}=5.4 \times 10^{3} \mathrm{MPa}$ for the brick, and $\lambda_{m}=1.1 \times 10^{3} \mathrm{MPa}$ and $\mu_{m}=1.6 \times 10^{3} \mathrm{MPa}$ for the mortar, respectively.

The interphase, localized at the brick/mortar interface level, is assumed to be a thin stratified layer comprising of brick and mortar material characteristics. In all numerical models proposed, the interphase is treated via the imperfect interface approach. In other words, it is a third material supposed to be initially-transversely isotropic, whose elastic constants $E_{1}, E_{3}, G_{13}$ and $v_{13}$ are derived starting from the mechanical properties of the constituents $\left(E_{b}, E_{m}, v_{b}, v_{m}\right)$. In order to obtain its elastic constants a preliminary standard homogenization for stratified is performed on the undamaged $\varepsilon$-thick representative elementary volume [48, 50, 51]. Moreover, this interphase is assumed to be microcracked.

As a result, the following effective elastic constants for the virgin interphase material are obtained: $E_{1}=8.5 \times 10^{3} \mathrm{MPa}, E_{3}=6.3 \times 10^{3} \mathrm{MPa}, G_{13}=5 \times 10^{3}$ MPa and $v_{13}=0.2$.

Two imperfect interface models are taken into account, the nonlinear St. VenantKirchhoff interface, and the soft interface model obtained in Sect. 2. Note that it is possible to applicate them in a three-dimensional context under the hypothesis of isotropic interface (i.e. the tangential interface stiffness is assumed to be isotropic in the interface plane). The nonlinear imperfect interface is modeled according Eq. (149):

$$
\mathbf{P i}_{3}=\frac{\hat{A}_{3333}}{2 \varepsilon^{2}}|[[\mathbf{u}]]|^{2}[[\mathbf{u}]]
$$

in which the interface stiffness $\hat{A}_{3333}$ is given by Eq. (148). Assuming the following values: $L=210 \mathrm{~mm}, l=L / 100=2 \mathrm{~mm}$ and $\varepsilon=0.2 \mathrm{~mm}$, the stiffness results in: $\hat{A}_{3333}=\frac{L}{2 B_{n n} l^{2}}=5.9 \times 10^{4} \mathrm{~N} / \mathrm{mm}^{3}$.

Concerning the linear-interface case, let the imperfect interface law be recalled:

$$
\boldsymbol{\sigma} \mathbf{i}_{3}=\mathbf{K}^{33}[[\mathbf{u}]]
$$


where the interface stiffnesses in normal and in tangential-to-the-interface directions comprised in the two-rank matrix $\mathbf{K}^{33}$, are expressed by Eq. (151):

$$
K_{T}=\frac{L}{B_{t t} l^{2}}, \quad K_{N}=\frac{L}{2 B_{n n} l^{2}}
$$

accordingly they result in: $K_{N}=5.9 \times 10^{4} \mathrm{~N} / \mathrm{mm}^{3}$ and $K_{T}=1.4 \times 10^{5} \mathrm{~N} / \mathrm{mm}^{3}$.

Several numerical simulations are carried out, aimed at validating the proposed interface models; in particular, in the model with the linearly elastic constituents, in what follows denoted as linear model, both the linear and the nonlinear interface laws, are implemented. In the nonlinear model, i.e. the one with the hyperelastic St. VenantKirchhoff constituents, only the nonlinear interface law is enforced. Additionally, the linear and the nonlinear models are also implemented with perfect interface condition (i.e. $[[\mathbf{u}]]=0,\left[\left[\sigma \mathbf{i}_{3}\right]\right]=0,\left[\left[\mathbf{P i}_{3}\right]\right]=0$ ), in order to have some reference data, in the following they are referred as LP and NLP, respectively. Moreover, in what follows the linear model with linear interface will be called $\mathrm{L}^{2}$, the linear model with nonlinear interface will be called LNL and the nonlinear model with nonlinear interface will be called $\mathrm{NL}^{2}$.

All analysis are performed with the software COMSOL Multiphysics ${ }^{\circledR} 4.3$ on a processor Intel(R) Core (TM) i3-2350M 2.3 GHz CPU. A free tetrahedral mesh of fine size is chosen in all the analysis cases for the whole domain, moreover, the brick/mortar interface is modeled through interface finite elements of zero thickness, as represented in Fig. 3. The implemented numerical models aim to reproduce a pushout test on a single brick in a quasi-static loading process. The tests are performed in displacement-controlled mode with an imposed displacement of a maximum value equal to $5 \mathrm{~mm}$. The degrees of freedom and the solution times expressed in seconds are summarized in Table 1, for all the analysis cases.

It is worth noting that it could be possible to reduce the degrees of freedom and, consequently, the CPU times, by applying some considerations about the geometrical symmetries of the considered system. Nevertheless, the remarkable aspect is the large difference in terms of CPU time among linear and nonlinear calculations, independently if the nonlinearity is localized at the interface level (LNL) or in the constituents $\left(\mathrm{NL}^{2}\right)$. Moreover, both in the linear and in the nonlinear model, the introduction of the linear and nonlinear-imperfect-interface conditions, i.e. $\mathrm{L}^{2}$ and

Table 1 Values of degrees of freedom (dof) and solution times (in seconds) for all the analyzed numerical models

\begin{tabular}{l|l|c}
\hline Model & dof & CPU time $(\mathrm{s})$ \\
\hline LP & $2,13,621$ & 309 \\
\hline $\mathrm{L}^{2}$ & $2,19,822$ & 326 \\
\hline $\mathrm{LNL}$ & $2,19,822$ & 9716 \\
\hline $\mathrm{NLP}$ & $2,13,621$ & 8831 \\
\hline $\mathrm{NL}^{2}$ & $2,19,822$ & 10,317 \\
\hline
\end{tabular}



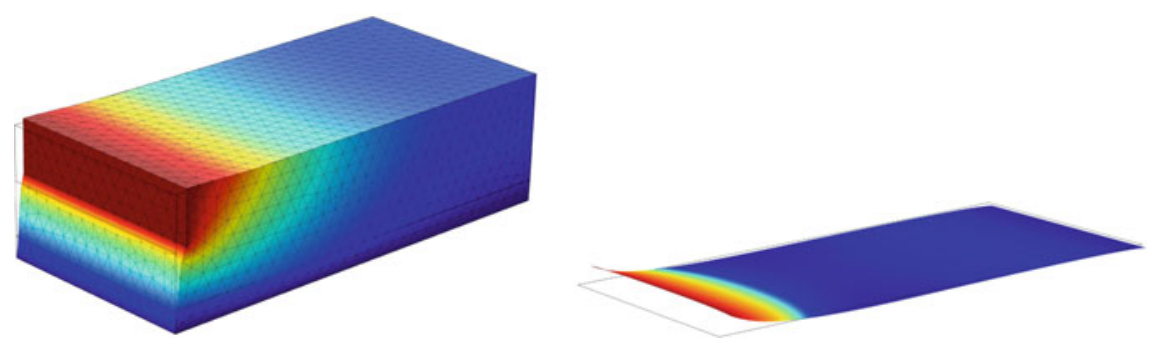

Fig. 4 Deformed shape in LNL model-final deformed shape relative to LNL model (on the right). The $x_{1}$-component of the displacement field is mapped in colors. Final deformed shape of the interface in the same model (on the left) with color map of the $x_{3}$-component of the displacementjump vector, the maximum value of the displacement is $0.12 \mathrm{~mm}$ (a factor scale of 5 is applied) (color online)

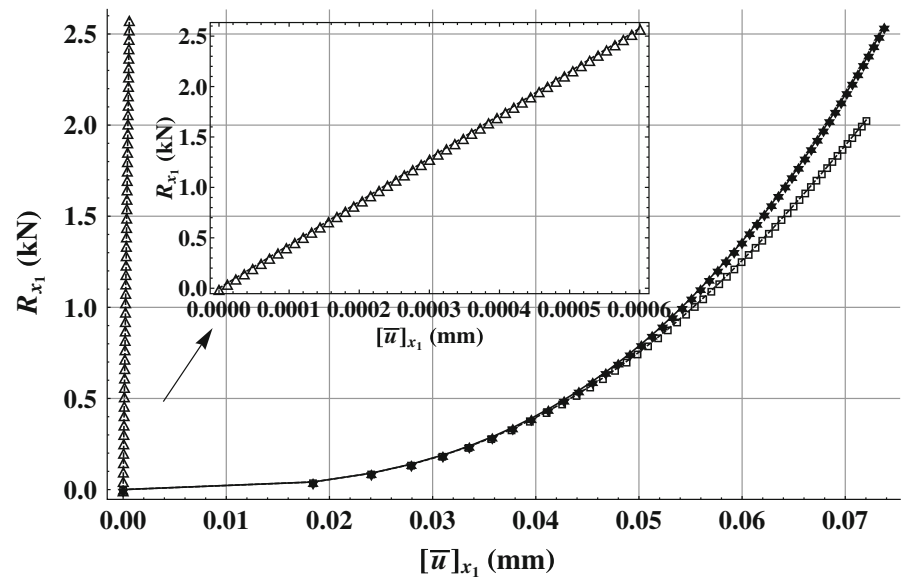

Fig. 5 Comparison in terms of reaction force-reaction force in the $x_{1}$-direction averaged over the loaded boundary surface versus the $x_{1}$-component of the average displacement-jump vector, at the final step (the maximum value of the imposed displacement is $5 \mathrm{~mm}$ ). Comparison among: $\mathrm{L}^{2}$

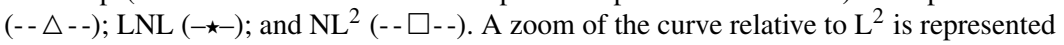

$\mathrm{NL}^{2}$ respectively, does not produce a significant increment of the CPU times with respect to the perfect-interface cases.

The numerical simulations stop when the imposed displacement reaches is maximum value $(5 \mathrm{~mm})$. In Fig. 4 a deformed shape at the final configuration is shown and the distribution of the displacement field is color-mapped.

The curves shown in Fig. 5 represent the $x_{1}$-component of the reaction force (i.e., in the acting direction of the imposed displacement) averaged on the loaded boundary, plotted with respect to the $x_{1}$-component of the displacement jump averaged over the interface surface, for all the analyzed cases. Interestingly, both models LNL and $\mathrm{NL}^{2}$, allow to take into account for larger deformations (about one order of magnitude) at the interface level, than the $\mathrm{L}^{2}$ model. 


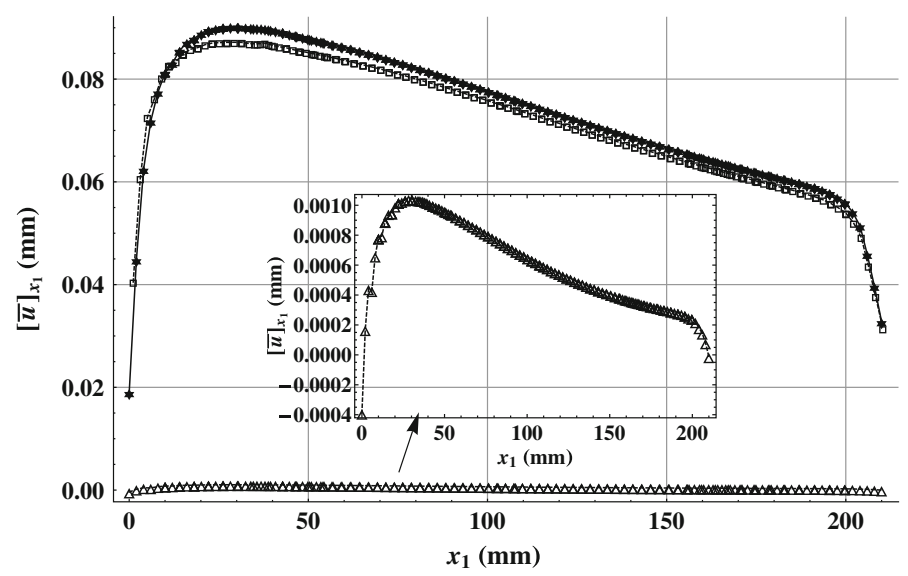

Fig. 6 Comparison in terms of displacement jump in $x_{1}$-direction-final distribution of the $x_{1}$-component of the average displacement-jump vector along the interface in the $x_{1}$-direction (recall that the maximum value of the imposed displacement is $5 \mathrm{~mm}$ ). Comparison among: $\mathrm{L}^{2}$

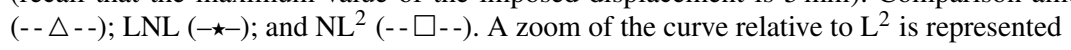

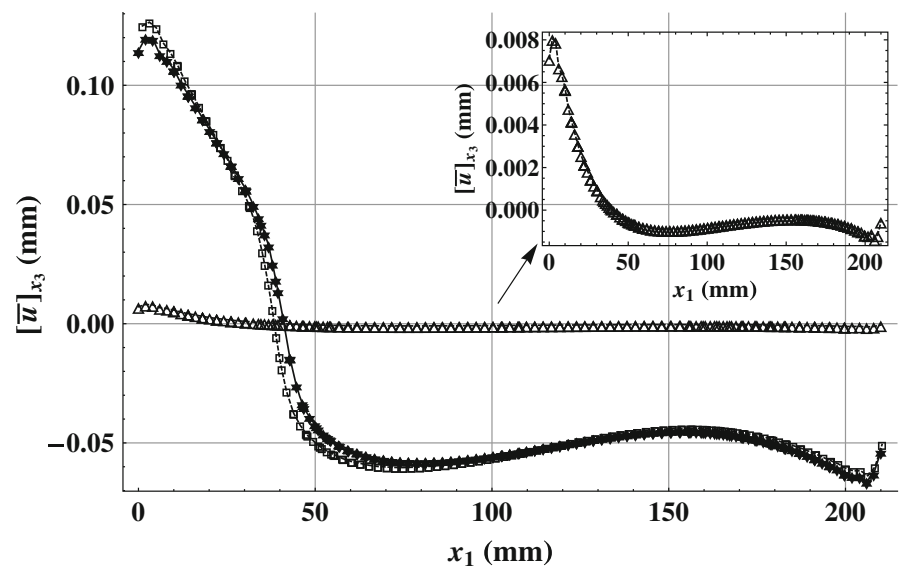

Fig. 7 Comparison in terms of displacement jump in $x_{3}$-direction-final distribution of the $x_{3}$-component of the average displacement-jump vector along the interface in the $x_{1}$-direction (recall that the maximum value of the imposed displacement is $5 \mathrm{~mm}$ ). Comparison among: $\mathrm{L}^{2}$

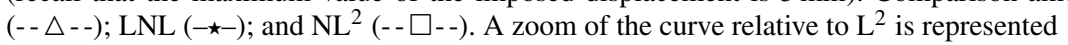

A comparison of Figs. 6 and 7 put in evidence this aspect. The figures represent the distribution, at the final configuration, of the $x_{1}$-component and of the $x_{3}$-component of the displacement-jump vector, respectively, along a cut line obtained from the intersection of the interface plane with the plane of symmetry.

Furthermore, Figs. 5, 6 and 7 highlight that is not very useful to model the adherents as hyperelastic materials in order to take into account the geometrical nonlinearities, i.e. large deformations, in terms of global response. In fact, the implementation 


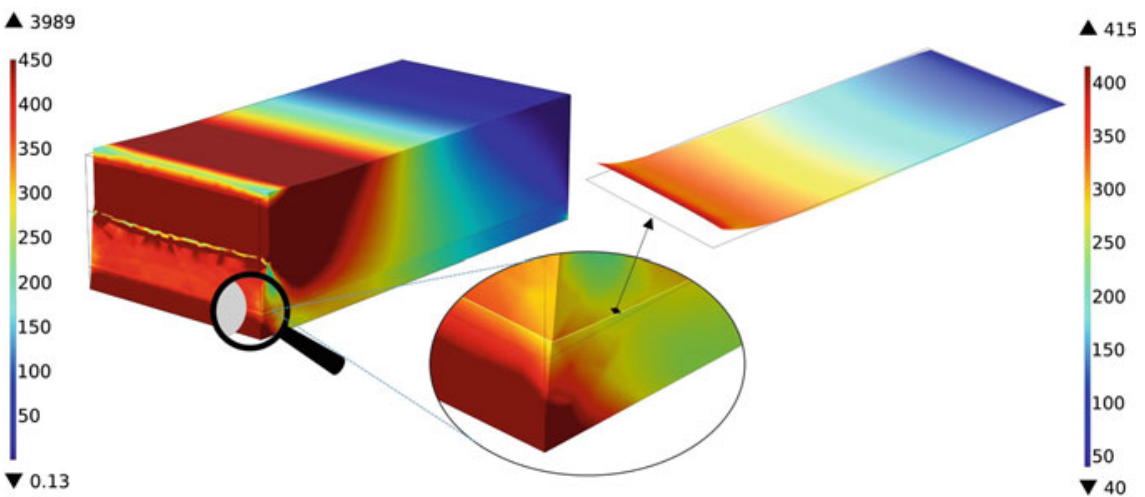

Fig. 8 Von Mises stresses in LNL model-Von Mises stress (MPa) in LNL, with a particular of the stress distribution at the interface level

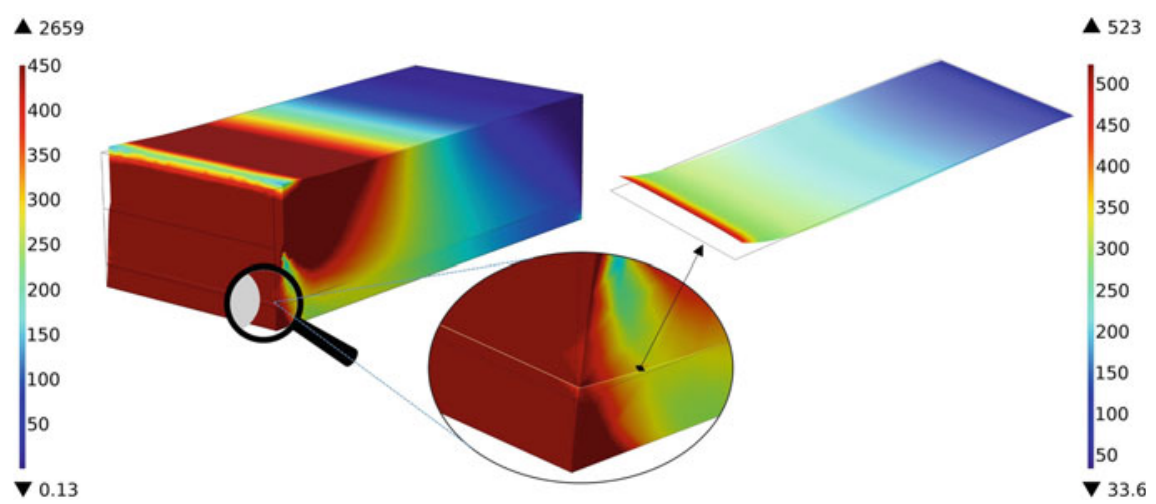

Fig. 9 Von Mises stresses in $\mathrm{NL}^{2}$ model-Von Mises stress (MPa) in $\mathrm{NL}^{2}$, with a particular of the stress distribution at the interface level

of a nonlinear imperfect interface, as the proposed St. Venant Kirchhoff model, in a linearly elastic composite system (LNL), seems to sufficiently catch the nonlinearinterface behavior as the fully nonlinear model $\left(\mathrm{NL}^{2}\right)$, reaching the same order of magnitude in terms of displacement jumps.

Figures 8 and 9 show the distribution of the Von Mises stresses in LNL and in $\mathrm{NL}^{2}$ respectively, in both cases a detail of the interface zone is represented. It is worth noting the difference in terms of magnitude of the stresses. In particular, in $\mathrm{NL}^{2}$ model the Von Mises stresses are significantly smaller than in LNL. Moreover, by analyzing the particular of the interfaces in both model, a significant difference in terms of stress distribution can be appreciated. 


\section{Conclusions}

In the first part of the present paper, the principal tools on which the imperfect interface approach is founded, have been introduced. After a brief recall on modeling background of imperfect interfaces, the matched asymptotic expansion method and the homogenization for microcracked media in NIA framework, have been extensively detailed.

The matched asymptotic expansion formulation, based on a higher order theory [53], has been formulated for both soft and hard interface cases. Thereby, the interface laws until the second (one) order have been derived, in both soft and hard interface conditions [53]. Such an asymptotic method, within the imperfect interface approach, is coupled to another tool, that is a micromechanical homogenization technique. In particular, a homogenization for microcracked media in the NIA framework has been chosen in order to take into account for damage in interphase. Two dual approaches in NIA have been presented, the stress-based and the strain-based approach.

In the hard imperfect interface model, the matched asymptotic technique has been expanded until the order one, within the higher order theory framework, recovering an imperfect interface law in terms of stresses and displacements jumps. This resulting interface law, is a challenging issue from a computational point of view. Moreover, a homogenization technique in the strain-based approach under the hypothesis of dilute concentration, is adopted [20, 21]. This homogenization technique leads to an expression of the effective elastic coefficients of the type: $f\left(\mathbb{C}_{0}\right)-d\left[g\left(\mathbb{C}_{0}\right)\right]$ with $f, g$ generic functions. From this expression, it is well highlighted that the values of density $d$ are severely limited. It is worth remarking that there exist other homogenization techniques in dilute concentration approximation that overcome this shortcoming, for instance the dilute estimate scheme by [6], for which the stiffness coefficients, in the initially-isotropic $\left(E^{0}, v^{0}\right)$ interphase case, are given by:

$$
\left\{\begin{aligned}
C_{\rho} & =\frac{3\left(2 v^{0}-1\right)+16\left(\left(1-v^{0}\right)^{2}\right) \rho}{3\left(2 v^{0}-1\right)+32\left(v^{0}\right)^{2}\left(1-v^{0}\right) \rho} \\
E_{1}^{1} & =C_{\rho} E^{0} \\
E_{3}^{1} & =E^{0} \\
\mu_{13}^{1} & =C_{\rho} \mu^{0} \\
v_{31}^{1} & =C_{\rho} v^{0}
\end{aligned}\right.
$$

Nevertheless, a great advantage of the Goidescu homogenization can be to lead to coefficients which do not depend on the REV geometry, because of the chosen form of the microstructural parameter $d$. For the St. Venant-Kirchhoff type interface (Sect. 3), a new matched asymptotic technique, based on fractional expansions of the relevant fields, has been proposed. This asymptotic procedure has been formulated by extending the asymptotic method to the finite strain theory [49, 54]. Also in this case, a homogenization has been performed to treat the microcracked interphase, i.e. the NIA building block in a stress-based approach.

Finally, a simple three-dimensional benchmark is proposed, in which three modeling cases have been compared in order to validate the proposed models. 
The chosen application domain is masonry. The first two models, defined as linear, have been conceived with linearly elastic adherents (brick and mortar), and with two different interface conditions. In the first case, the brick/mortar interface has been modeled with the linear spring-type interface law, and in the second case, the St. Venant-Kirchhoff nonlinear interface law has been implemented. The third model, defined as nonlinear, is a fully nonlinear one, in which the adherents are assumed to be St. Venant-Kirchhoff hyperelastic material and the interface has been modeled with the St. Venant-Kirchhoff nonlinear interface law. Some comparisons have been carried out in terms of displacement jumps and of stresses distribution along the interface. The soundness and the consistency of the proposed interface models are highlighted, both from a theoretical and a numerical points of view. Moreover, it has been established that the linear model with the nonlinear interface is able to catch the large displacements occurring at the interface level as much as the fully nonlinear model, additionally, the computational cost in the first case is smaller.

\section{References}

1. R. Abdelmoula, M. Coutris, and J.J. Marigo. Asymptotic behavior of an elastic thin layer. C. R. Acad. Sci.. Serie II F. B-Mec. Phys. Astr., 326(4):237-242, 1998.

2. S. ANDRIEUX, Y. BAMBERGER, AND J.J. MARIGO. A model of micro-cracked material for concretes and rocks. J. Méc. Theor. Appl., 5(3):471-513, 1986.

3. Y. BENVENISTE. The effective mechanical behaviour of composite materials with imperfect contact between the constituents. Mech. Mat., 4(2):197-208, 1985.

4. Y. BENVENISTE. A general interface model for a three-dimensional curved thin anisotropic interphase between two anisotropic media. J. Mech. Phys. Solid, 54(4):708-734, 2006.

5. Y. BENVENISTE AND T. MILOH. Imperfect soft and stiff interfaces in two-dimensional elasticity. Mech. Mat., 33(6):309-323, 2001.

6. P.G. Bornert, T. BRetheAU, AND P. GILORMini. Homogénéisation en mécanique des matériaux, Tome 1 : Matériaux aléatoires élastiques et milieux périodiques. Hermes Sciences, Paris, 2001.

7. J.R. BRISTOW. Microcracks, and the Static and Dynamic Elastic Constants of Annealed and Heavily Cold-worked Metals. Br. J. Appl. Phys., 11(2):81-85, 1960.

8. B. BUDIANSKY AND R.J. O'CONNEL. Elastic moduli of a cracked solid. Int. J. Solid Struct., 12:81-97, 1976.

9. P. BOVIK. On the modelling of thin interface layers in elastic and acoustic scattering problems. Quart. J. Mech. Appl. Math., 47(1):17-42, 1994.

10. D. CAILLERIE. Behavior at limit of a thin inclusion of high stiffness in an elastic body. $C$. R. Hebd. Seances Acad. Sci. Serie A, 287(8):675-678, 1978.

11. P.G. CIARLET. Recent Progress in the Two-Dimensional Approximation of Three - Dimensional Plate Models in Nonlinear Elasticity. In EDUARDO L. ORTIZ, editor, Numerical Approximation of Partial Differential Equations Selection of Papers Presented at the International Symposium on Numerical Analysis held at the Polytechnic University of Madrid, 133 of North-Holland Mathematics Studies, pages 3-19. North-Holland, 1987.

12. P.G. Ciarlet. Mathematical Elasticity. In PhilipPe G. Ciarlet, editor, Mathematical Elasticity Volume II: Theory of Plates, 27 of Studies in Mathematics and Its Applications, pages vii-xi. Elsevier, 1997.

13. P.G. Ciarlet AND P. DeStuYnder. A justification of a nonlinear model in plate theory. Comput. Meth. Appl. Mech. Eng., 17-18, Part 1(0):227-258, 1979. 
14. S. Dumont, F. Lebon, AND R. Rizzoni. An asymptotic approach to the adhesion of thin stiff films. Mech. Res. Commun., 58(0):24-35, 2014.

15. S. Dumont, F. LEBOn, M.L. RAFFA, AND R. RIZZONI. Towards nonlinear imperfect interface models including micro-cracks and smooth roughness. Annal Solid Struct. Mech., In press.

16. J.D. ESHELBY. Elastic inclusions and inhomogeneities. In Progr. Solid mech., 2, page 87-140. North-Holland, Amsterdam, 1961.

17. F. Fouchal, F. Lebon, M.L. RAFFa, and G. Vairo. An interface model including cracks and roughness applied to masonry. Open Civ. Eng. J., 8:263-271, 2014.

18. G. GEYMONAT AND F. KRASUCKI. Analyse asymptotique du comportement en flexion de deux plaques collées. C. R. Acad. Sci. - Series IIB - Mech.-Phys.-Chem.-Astr., 325(6):307-314, 1997.

19. Y. Gilibert AND A. Rigolot. Asymptotic analysis of double adhesive bonded joints loaded in shear tension (in French). J. Méc. Appl., 3(3):341-372, 1979.

20. C. GoIDESCU. Caractérisation et modélisation de l'endommagement par microfissuration des composites stratifiés - Apports des mesures de champs et de l'homogénéisation. $\mathrm{PhD}$ thesis, Institut National Polytechnique de Toulouse (INP Toulouse), 2011.

21. C. Goidescu, H. Welemane, D. Kondo, and C. Gruescu. Microcracks closure effects in initially orthotropic materials. Eur. J. Mech. A-Solid, 37:172-184, 2013.

22. M. Goland AND E. ReISSNER. The stresses in cemented joints. J. Appl. Mech., 11: A17-A27, 1944.

23. S.T. GU AND Q.C. HE. Interfacial discontinuity relations for coupled multifield phenomena and their application to the modeling of thin interphases as imperfect interfaces. $J$. Mech. Phys. Solid, 59(7):1413-1426, 2011.

24. S.T. Gu. Contributions to the modelling of imperfect interfaces and to the homogenization of heterogeneous materials. Ph.D. Thesis (in French). PhD thesis, Université Paris-Est Marne-laVallée, France, 2008.

25. Z. HASHIN. The differential scheme and its application to cracked materials. J. Mech. Phys. Solid, 36(6):719-734, 1988.

26. Z. HASHIN. Thermoelastic properties of fiber composites with imperfect interface. Mech. Mat., 8(4):333-348, 1990.

27. Z. HASHIN. Thermoelastic properties of particulate composites with imperfect interface. J. Mech. Phys. Solid, 39(6):745-762, 1991.

28. Z. HASHIN. The Spherical Inclusion With Imperfect Interface. J. Appl. Mech., 58(2): 444-449, June 1991.

29. Z. HASHIN. Extremum principles for elastic heterogenous media with imperfect interfaces and their application to bounding of effective moduli. J. Mech. Phys. Solid, 40(4):76-781, 1992.

30. Z. HASHIN. Thin interphase/imperfect interface in elasticity with application to coated fiber composites. J. Mech. Phys. Solid, 50(12):2509-2537, 2002.

31. R. HILL. Elastic Properties of Reinforced Solids - Some Theoretical Principles. J. Mech. Phys. Solid, 11(5):357-372, 1963.

32. R. HILl. A Self-consistent Mechanics of Composite Materials. J. Mech. Phys. Solid, 13(4):213-222, 1965.

33. H. HORII AND S. NEMAT- NASSER. Overall moduli of solids with microcracks: Loadinduced anisotropy. J. Mech. Phys. Solid, 31(2):155-171, 1983.

34. M. KACHANOV. Elastic solids with many cracks and related problems. Adv. Appl. Mech., 30:259-445, 1994.

35. M. KACHANOV AND I. SEVOSTIANOV. On quantitative characterization of microstructures and effective properties. Int. J. Solid Struct., 42(2):309-336, 2005.

36. A. KLARBRING. Derivation of a model of adhesively bonded joints by the asymptotic expansion method. Int. J. Eng. Sci., 29(4):493-512, 1991.

37. A. Klarbring and A.B. Movchan. Asymptotic modelling of adhesive joints. Mech. Mat., 28(1-4):137-145, 1998. 
38. F. Lebon AND R. RIZZONI. Asymptotic analysis of a thin interface: The case involving similar rigidity. Int. J. Eng. Sci., 48(5):473-486, 2010.

39. F. LEBON AND R. RIZZONI. Asymptotic behavior of a hard thin linear elastic interphase: An energy approach. Int. J. Solid Struct., 48(3-4):441-449, 2011.

40. F. LEBON AND F. ZAITTOUNI. Asymptotic modelling of interfaces taking contact conditions into account: Asymptotic expansions and numerical implementation. Int. J. Eng. Sci., 48(2):111-127, 2010.

41. F. Lebon, A. OUlD KhaOUA, AND C. LiCht. Numerical study of soft adhesively bonded joints in finite elasticity. Computat. Mech., 21:134-140, 1998.

42. F. LEBON, R. RIZZONI, AND S. RONEL- IDRISSI. Asymptotic analysis of some non-linear soft thin layers. Comput. \& Struct., 82(23-26):1929-1938, 2004.

43. C. MAUGE AND M. KACHANOV. Effective elastic properties of an anisotropic material with arbitrarily oriented interacting cracks. J. Mech. Phys. Solid, 42(4):561-584, 1994.

44. A. NEEDLEMAN. An analysis of decohesion along an imperfect interface. Int. J. Fract., 42(1):21-40, 1990.

45. A. NEEdLEMAN. Micromechanical modelling of interfacial decohesion. Ultramicroscopy, 40(3):203-214, 1992.

46. A. OUlD KhaOUA. Etude théorique et numérique de problemes de couches minces en elasticité. PhD thesis, Université de Montpellier Sciences et Techniques du Languedoc, 1995.

47. P. PONTE CASTAÑEDA AND J.R. WILLIS. The effect of spatial distribution on the effective behavior of composite materials and cracked media. J. Mech. Phys. Solid, 43(12):19191951, 1995.

48. M.L. RafFA, F. Lebon, E. SACCO, AND H. Welemane. A multi-level interface model for damaged masonry. In B.H.V. Topping, P. Iványi, (Editors), "Proceedings of the Fourteenth International Conference on Civil, Structural and Environmental Engineering Computing”, Civil-Comp Press, Stirlingshire, UK, Paper 64, 2013., 2013.

49. M.L. RAFFA, F. LEBON, AND R. RIZZONI. On modeling brick/mortar interface via a St. Venant-Kirchhoff orthotropic soft interface. Part I: theory. Int. J. Mason. Res. Innov., In press.

50. A. REKIK AND F. LEBON. Identification of the representative crack length evolution in a multi-level interface model for quasi-brittle masonry. Int. J. Solid Struct., 47(22-23): 3011-3021, 2010.

51. A. REKIK AND F. LEBON. Homogenization methods for interface modeling in damaged masonry. Adv. Eng. Softw., 46(1):35-42, 2012.

52. R. RIZZONI AND F. LEBON. Imperfect interfaces as asymptotic models of thin curved elastic adhesive interphases. Mech. Res. Commun., 51(0):39-50, 2013.

53. R. Rizzoni, S. Dumont, F. LEBon, AND E. SACCO. Higher order model for soft and hard elastic interfaces. Int. J. Solid Struct., 51(23-24):4137-4148, 2014.

54. R. RIZZONI, DUMONT S., AND F. LEBON. On Saint Venant - Kirchhoff Imperfect Interfaces. Submitted.

55. E. SANCHEZ- PALENCIA. Non-homogenous media and vibration theory. Lecture notes in physics. Springer-Verlag, Berlin; New York, 1980.

56. E. SANCHEZ-PALENCIA and J. SANCHEZ-HUBERT. Introduction aux méthodes aymptotiques et à l' homogénéisation. Masson, 1992.

57. I. Sevostianov and M. Kachanov. Non-interaction Approximation in the Problem of Effective Properties. In MARK KACHANOV AND IGOR SEVOSTIANOV, editors, Effective Properties of Heterogeneous Materials, 193 of Solid Mechanics and Its Applications, pages 1-95. Springer Netherlands, 2013.

58. J.J. TELEGA. Homogenization of fissured elastic solids in the presence of unilateral conditions and friction. Computat. Mech., 6(2):109-127, 1990.

59. I. TSUKROV AND M. KACHANOV. Anisotropic material with arbitrarily oriented cracks and elliptical holes: Effective elastic moduli. Int. J. Fract., 92(1):L9-L14, 1998.

60. I. TSUKROV AND M. KACHANOV. Effective moduli of an anisotropic material with elliptical holes of arbitrary orientational distribution. Int. J. Solid Struct., 37(41):5919-5941, 2000 . 